\title{
The Ciliopathy Gene Ftm/Rpgrip1l Controls Mouse Forebrain Patterning via Region-Specific Modulation of Hedgehog/Gli Signaling
}

\author{
Abraham Andreu-Cervera, Isabelle Anselme, Alice Karam, Christine Laclef, Martin Catala, \\ and DSylvie Schneider-Maunoury \\ Sorbonne Université, Centre National de la Recherche Scientifique (CNRS) UMR7622, Institut national pour la Santé et la Recherche Médicale U1156, \\ Institut de Biologie Paris Seine-Laboratoire de Biologie du Développement (IBPS-LBD), 75005 Paris, France
}

Primary cilia are essential for CNS development. In the mouse, they play a critical role in patterning the spinal cord and telencephalon via the regulation of Hedgehog/Gli signaling. However, despite the frequent disruption of this signaling pathway in human forebrain malformations, the role of primary cilia in forebrain morphogenesis has been little investigated outside the telencephalon. Here we studied development of the diencephalon, hypothalamus and eyes in mutant mice in which the Ftm/Rpgrip1l ciliopathy gene is disrupted. At the end of gestation, $\mathrm{Ftm}^{-1-}$ fetuses displayed anophthalmia, a reduction of the ventral hypothalamus and a disorganization of diencephalic nuclei and axonal tracts. In $\mathrm{Ftm}^{-1-}$ embryos, we found that the ventral forebrain structures and the rostral thalamus were missing. Optic vesicles formed but lacked the optic cups. In $\mathrm{Ftm}^{-1-}$ embryos, Sonic hedgehog (Shh) expression was virtually lost in the ventral forebrain but maintained in the zona limitans intrathalamica (ZLI), the mid-diencephalic organizer. Gli activity was severely downregulated but not lost in the ventral forebrain and in regions adjacent to the Shh-expressing ZLI. Reintroduction of the repressor form of Gli3 into the $\mathrm{Ftm}^{-1-}$ background restored optic cup formation. Our data thus uncover a complex role of cilia in development of the diencephalon, hypothalamus and eyes via the region-specific control of the ratio of activator and repressor forms of the Gli transcription factors. They call for a closer examination of forebrain defects in severe ciliopathies and for a search for ciliopathy genes as modifiers in other human conditions with forebrain defects.

Key words: ciliopathy; forebrain patterning; Hedgehog/Gli signaling; primary cilia; Rpgrip1l

Significance Statement

The Hedgehog $(\mathrm{Hh})$ signaling pathway is essential for proper forebrain development as illustrated by a human condition called holoprosencephaly. The Hh pathway relies on primary cilia, cellular organelles that receive and transduce extracellular signals and whose dysfunctions lead to rare inherited diseases called ciliopathies. To date, the role of cilia in the forebrain has been poorly studied outside the telencephalon. In this paper we study the role of the Ftm/Rpgrip1l ciliopathy gene in mouse forebrain development. We uncover complex functions of primary cilia in forebrain morphogenesis through region-specific modulation of the Hh pathway. Our data call for further examination of forebrain defects in ciliopathies and for a search for ciliopathy genes as modifiers in human conditions affecting forebrain development.

\section{Introduction}

The Hedgehog (Hh) pathway plays an essential role in forebrain patterning, as illustrated by its frequent perturbation in holo- prosencephaly (HPE), a human condition defined as a defect in the formation of midline structures of the forebrain and face

tine Vesque and Marie Breau (IBPS-LBD) and Christoph Gerhardt (Institute for Animal Developmental and Molecular Biology, Heinrich Heine University Düsseldorf, Germany) for critical reading of the paper, and James Briscoe (Crick Institute, London, UK) for the kind gift of the Tg[GBS::GFP] transgenic line.

The authors declare no competing financial interests.

Correspondence should be addressed to Sylvie Schneider-Maunoury at sylvie.schneider-maunoury@upmc.fr. https://doi.org/10.1523/JNEUROSCI.2199-18.2019

Copyright $\odot 2019$ Andreu-Cervera et al.

This is an open-access article distributed under the terms of the Creative Commons Attribution License Creative Commons Attribution 4.0 International, which permits unrestricted use, distribution and reproduction in any medium provided that the original work is properly attributed. 
(Muenke and Beachy, 2001; Fernandes and Hébert, 2008). Null mouse mutants for Sonic hedgehog (Shh) display a HPE phenotype (Chiang et al., 1996) and studies involving gene inactivation in mouse, lineage tracing, and loss- and gain-of-function approaches in chick identified multiple, successive functions of the Hh pathway in the diencephalon, hypothalamus, and eyes (Furimsky and Wallace, 2006; Vue et al., 2009; Jeong et al., 2011; Alvarez-Bolado et al., 2012; Haddad-Tóvolli et al., 2012, 2015; Blaess et al., 2015; Zhang and Alvarez-Bolado, 2016).

In vertebrates, transduction of $\mathrm{Hh} / \mathrm{Gli}$ signaling depends on primary cilia, microtubular organelles with sensory functions. In the developing CNS, primary cilia are essential for proper dorsoventral (DV) patterning of the spinal cord via modulating $\mathrm{Hh}$ signaling. Shh binds to its receptor Ptch1, which removes Ptch1 from the cilium and relieves the inhibition of the G-proteincoupled receptor Smoothened (Smo) by Ptch1. Hh signaling at the cilium leads to the translocation of the Gli transcription factors into the nucleus and their activation into Gli activator form (GliA). In the absence of ligand, Gli2 and Gli3 are targeted to the proteasome in a cilium-dependent manner, giving rise to short forms with transcriptional repressor activity, among which Gli3R is a particularly strong repressor. Thus, the primary cilium is essential for the production of both GliR and GliA forms (Goetz and Anderson, 2010). In the forebrain, functional primary cilia are required for correct DV patterning of the telencephalon (Willaredt et al., 2008; Stottmann et al., 2009; Besse et al., 2011; Benadiba et al., 2012; Willaredt et al., 2013; Laclef et al., 2015) and for the proliferation of granule cell precursors in the dentate gyrus (Han et al., 2008). Surprisingly, despite the essential function of Hh signaling in the forebrain, the role of primary cilia outside the telencephalon has been little explored (Willaredt et al., 2013).

In this paper we study the function of the Ftm/Rpgripllgene in the forebrain. RPGRIP1L is a causal gene in severe human ciliopathies with brain abnormalities, Meckel-Gruber syndrome (MKS5 OMIM 611561) and Joubert syndrome type B (JBTS7 OMIM 611560; Arts et al., 2007; Delous et al., 2007). The Rpgrip1l protein is enriched at the ciliary transition zone (TZ), a region at the base of the axoneme distal to the basal body involved in the control of ciliary protein entry and exit (Reiter et al., 2012). Rpgrip1l is essential for the TZ localization of many other ciliopathy proteins (Mahuzier et al., 2012; Reiter et al., 2012; Shi et al., 2017; Wiegering et al., 2018). Rpgrip1l is also required for proteasome activity at the cilium base and for autophagy (Gerhardt et al., 2015; Struchtrup et al., 2018).

$\mathrm{Ftm}^{-1-}$ mouse fetuses die at or shortly before birth with a ciliopathy phenotype (Delous et al., 2007; Vierkotten et al., 2007) and lack cilia in the developing telencephalon (Besse et al., 2011). Using this mutant, our laboratory has previously shown that primary cilia are required for telencephalic DV patterning. In $\mathrm{Ftm}^{-1-}$ embryos, the olfactory bulbs and corpus callosum, two dorsal telencephalic structures, are missing because of an expansion of the ventral telencephalon. The phenotype is rescued by introduction into the Ftm mutant of one allele of Gli3 ${ }^{\Delta 699}$ (Besse et al., 2011; Laclef et al., 2015), which produces constitutively a short form of Gli3 with repressor activity (Hill et al., 2007). These studies demonstrate that the main role of cilia in telencephalic patterning is to permit Gli3R formation.

What is the role of primary cilia in other forebrain regions? Here we show that $\mathrm{Ftm}^{-1-}$ fetuses display severely disorganized hypothalamus and diencephalon and lack eyes. Investigating the molecular causes of these defects, we find that $S h h$ expression and Hh signaling (hereafter called Shh signaling, Shh being the prin- cipal ligand in the forebrain) are differentially affected in different forebrain regions. Our results uncover essential and diverse functions for Ftm/Rpgrip1l and cilia in Gli activity in patterning the forebrain and eyes.

\section{Materials and Methods}

Mice. All experimental procedures involving mice were made in agreement with the European Directive 2010/63/EU on the protection of animals used for scientific purposes, and the French application decree 2013-118. Mice were raised and maintained in the IBPS mouse facility, approved by the French Service for Animal Protection and Health, with the approval numbers C-75-05-24. The project itself has been approved by the local ethical committee "Comité d'éthique Charles Darwin", under the authorization \#2015052909185846. Gli3 ${ }^{\Delta 699}$ - also named Gli3 ${ }^{\Delta}$ and Ftm-deficient mice were produced and genotyped as described previously (Böse et al., 2002; Besse et al., 2011). Mutant lines were maintained as heterozygous $\left(\mathrm{Ftm}^{+/-}\right.$or $\mathrm{Gli}^{\mathbf{\Delta} 699 /+}$ ) and double-heterozygous $\left(\mathrm{Ftm}^{+/-} ; \mathrm{Gli3}^{\mathbf{\Delta 6 9 9 / +}}\right.$ ) animals in the $\mathrm{C} 57 \mathrm{BL} / 6 \mathrm{~J}$ background. Note that the eye phenotype of the $\mathrm{Ftm}^{-1-}$ animals was totally penetrant in the C57BL/6J background used here, unlike in $\mathrm{C} 3 \mathrm{H}$ or mixed backgrounds (Delous et al., 2007; Wiegering et al., 2018). The transgenic line Tg[GBS:: GFP] was maintained in the C57BL/6J background and genotyped as described previously (Balaskas et al., 2012). In analyses of Ftm mutant phenotypes, heterozygous and wild-type (WT) embryos did not show qualitative differences, and both were used as "control" embryos. The sex of the embryos and fetuses was not analyzed. Embryonic day (E) 0.5 was defined as noon on the day of vaginal plug detection.

Histology, ISH, and IF. For whole-mount in situ hybridization (ISH), embryos were dissected in cold PBS and fixed in 4\% paraformaldehyde (PFA) in PBS for a time depending on the embryonic age and then processed as described by Anselme et al. (2007). For histology and ISH on sections, embryos were dissected in cold PBS and fixed overnight in $60 \%$ ethanol, 30\% formaldehyde and 10\% acetic acid. Embryos were embedded in paraffin and sectioned $(7 \mu \mathrm{m})$. Cresyl thionin staining and ISH were performed on serial sections, as described previously (Anselme et al., 2007; Besse et al., 2011; Laclef et al., 2015). For fluorescence ISH (FISH), immunodetection of the probe was done overnight at $4^{\circ} \mathrm{C}$ with anti-Digoxigenin peroxidase-conjugated antibody (Roche), diluted 1:50 in maleate buffer supplemented with $2 \%$ Boehringer Blocking Reagent (Roche). Peroxidase activity was detected with FITC-coupled tyramide (1:50).

For immunofluorescence (IF), embryos were fixed overnight in $4 \%$ PFA. E18.5 fetuses were perfused with $4 \%$ PFA. IF staining was performed on $14 \mu \mathrm{m}$ serial cryostat sections, as described previously (Anselme et al., 2007; Laclef et al., 2015), with antibodies against Shh (Cell Signaling Technology, 2207; 1:200 and R\&D Systems, AF445; 1:200), Arl13b (Neuromab 75-287; 1:1500), FoxA2 (Abcam, ab23630; 1:200), GFP (Aves, GFP-1020; 1:200), Rpgrip1l (Besse et al., 2011; 1:800), Mash1 (BD Pharmigen, 556604; 1:200), Neurofilament [Developmental Studies Hybridoma Bank (DSHB) 2H3; 1:200], Tuj1 (Sigma-Aldrich, T8578; 1:500), Tag1 (DSHB, 23.4-5; 1:50), and Robo3 (R\&D Systems, AF3076; 1:200). Secondary antibodies were AlexaFluor conjugates from Invitrogen (1:1000). Nuclei were stained with DAPI (1:500).

DiI/DiA labeling. Brains of E18.5 fetuses were dissected in PBS $1 \times$ and fixed overnight in $4 \%$ PFA. After three washes in PBS, brains were labeled by $1,1^{\prime}$-dioctadecyl-3,3,3',3'-tetramethylindocarbocyanine perchlorate (DiI; Invitrogen D383) or 4-Di-16 ASP [4,4-(dihexadecylaminostyril)$\mathrm{N}$-methylpyridinium iodide (DiA); Invitrogen, D3883] crystals, in the cortex or in the diencephalon of control and $\mathrm{Ftm}^{-1-}$ brains, as indicated in Figure 1. Samples were kept for at least 2 weeks in PFA $4 \%$ at $37^{\circ} \mathrm{C}$ for the lipophilic dye to diffuse along the fixed cell membranes. Then, the brains were embedded in $4 \%$ agarose in PBS, and thick coronal vibratome (LeicaVT1000S) sections were made.

Image acquisition and quantification of fluorescence intensity. ISH images were acquired with a bright-field Leica MZ16 stereomicroscope. IF, FISH, and axonal tract dye labeling images were observed with a fluorescent binocular (Leica, M165FC) and acquired with a confocal microscope (Leica, TCS SP5 AOBS). 

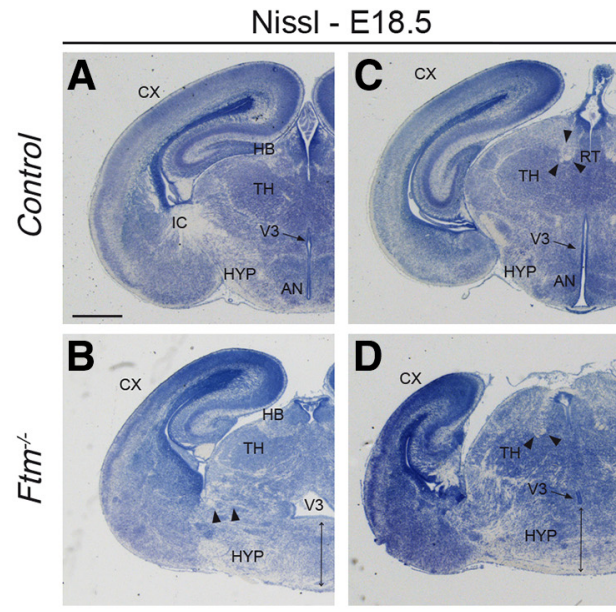

Tuj1/NF/DAPI - E13.5
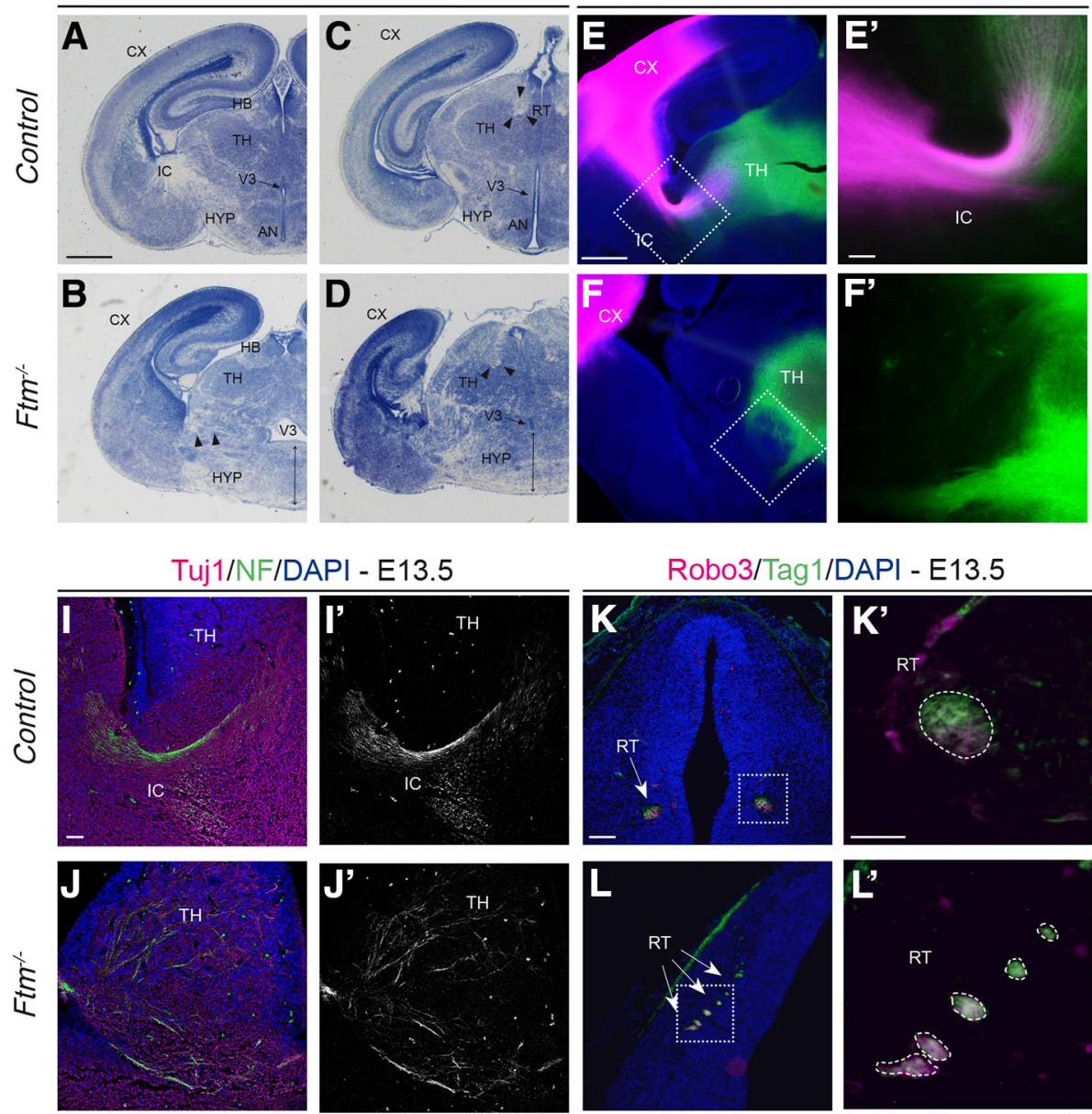

Robo3/Tag1/DAPI - E13.5
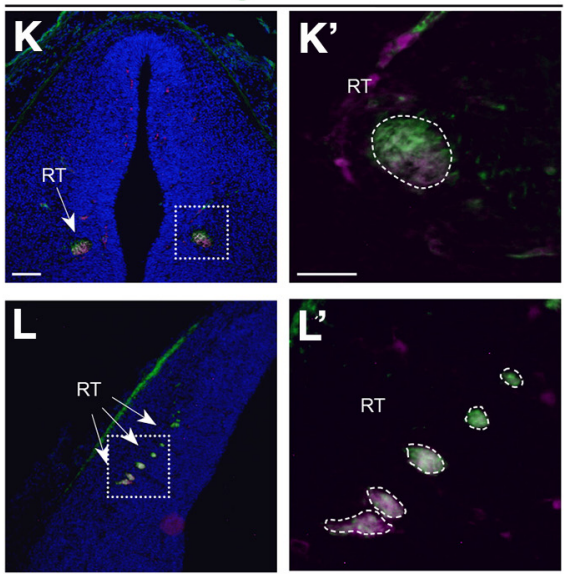

NF/DAPI - E18.5
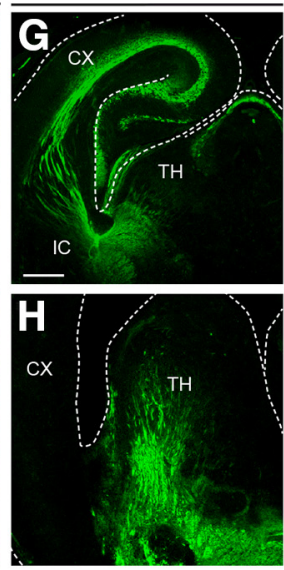
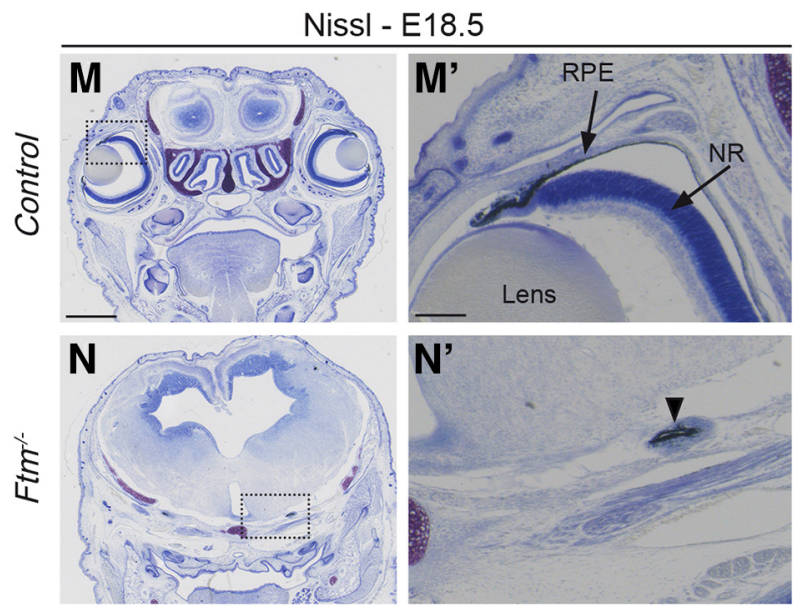

Figure 1. Histology and labeling of axon tracts in the brain of control and $\mathrm{Ftm}^{-1-}$ fetuses. $\boldsymbol{A}-\boldsymbol{D}$, Nissl staining on coronal sections of the brain at two distinct anteroposterior levels of thalamic and hypothalamic regions in E18.5 WT $(\boldsymbol{A}, \boldsymbol{C})$ and $\mathrm{Ftm}^{-/-}(\boldsymbol{B}, \boldsymbol{D})$ fetuses. $\boldsymbol{C}, \boldsymbol{D}$, More posterior sections than $\boldsymbol{A}$ and $\boldsymbol{B}$. Both levels of sections correspond to the ventral hypothalamus and the alar thalamus. Black arrowheads in $\boldsymbol{B}-\boldsymbol{D}$ point to axon fascicles of the IC and RT. Double black arrows in $\boldsymbol{B}$ and $\boldsymbol{D}$ point to the dysmorphic hypothalamus in $F$ tm mutants. $\boldsymbol{E}-\boldsymbol{F}^{\prime}$, Carbocyanine dye staining of corticothalamic (Dil, magenta) and thalamocortical (DiA, green) axons in E18.5 WT $\left(\boldsymbol{E}, \boldsymbol{E}^{\prime}\right)$ and $F t m^{-1-}\left(\boldsymbol{F}, \boldsymbol{F}^{\prime}\right)$ brains. $\boldsymbol{E}^{\prime}, \boldsymbol{F}^{\prime}$, Higher-magnification of the boxed regions in $\boldsymbol{E}$ and $\boldsymbol{F}$, respectively. $\boldsymbol{G}, \boldsymbol{H}$, Neurofilament (NF) immunostaining of axon tracts in E18.5 control $(\mathbf{G})$ and $F \mathrm{Fm}^{-/-}(\boldsymbol{H})$ brains. $\boldsymbol{I}-\boldsymbol{L}^{\prime}$, Immunofluorescence for Tuj1 and NF $\left(\boldsymbol{I}-\boldsymbol{J}^{\prime}\right)$ and for Rob03 and Tag1 $\left(\boldsymbol{K}-\boldsymbol{L}^{\prime}\right)$ in E13.5 control $\left(\boldsymbol{K}, \boldsymbol{K}^{\prime}\right)$ and $F t m^{-1-}\left(\boldsymbol{L}, \boldsymbol{L}^{\prime}\right)$ brains. $\boldsymbol{M}-\boldsymbol{N}^{\prime}$, Nissl staining on coronal sections at the level of the eyes of the head ofE18.5 WT $\left(\boldsymbol{M}, \boldsymbol{M}^{\prime}\right)$ and $F t m^{-1-}\left(\boldsymbol{N}, \boldsymbol{N}^{\prime}\right)$ fetuses. $\boldsymbol{M}^{\prime}, \boldsymbol{N}^{\prime}$, Higher-magnification of the boxed regions in $M$ and $N$, respectively. The arrowhead in $N^{\prime}$ points to remnants of the RPE. AN, Anteroventral nucleus; $C X$, cortex; HB, Habenula; NR, neural retina; $V 3$ third ventricle. Scale bars: (in $\boldsymbol{A}, \boldsymbol{M}$ ) $\boldsymbol{A}-\boldsymbol{D}, \boldsymbol{M}, \boldsymbol{N}, 1 \mathrm{~mm}$; (in $\boldsymbol{E}, \boldsymbol{G}) \boldsymbol{E}-\boldsymbol{H}, 0.5 \mathrm{~mm}$; (in $\left.\boldsymbol{E}^{\prime}, \boldsymbol{I}, \boldsymbol{K}^{\prime}\right), \boldsymbol{E}^{\prime}, \boldsymbol{F}^{\prime}, \boldsymbol{I}-\boldsymbol{J}^{\prime}, \boldsymbol{K}^{\prime}, \boldsymbol{L}^{\prime}, 0.1 \mathrm{~mm}$; (in $\boldsymbol{K}, \boldsymbol{M}^{\prime}$ ) $\boldsymbol{K}, \boldsymbol{L}, \boldsymbol{M}^{\prime}, \boldsymbol{N}^{\prime}, 0.2 \mathrm{~mm}$.

Fluorescence intensity was measured using ImageJ software. For ShhGFP immunofluorescence, adjacent squares of $50 \mu \mathrm{m}$ side were drawn in the diencephalon, all along the ventricular surface from posterior to anterior. Total fluorescence intensity was measured in each square on three distinct optical sections. For each optical section, the background intensity was measured by taking three squares in the third ventricle, and the mean background intensity was subtracted from all the measurements of the same image. Images from three controls, three $\mathrm{Ftm}^{-1-}$, and two $\left[\mathrm{Ftm}^{-1-}, \mathrm{Gli3}^{\mathrm{\Delta /+}}\right]$ embryos were used for quantification. For comparison, the measurements were aligned using as a reference the square 
corresponding to the AP level of the zona limitans intrathalamica (ZLI; point 6 of the ordinate in Fig. $7 P, Q)$. The diagrams in Fig. $7 P, Q$ indicate the mean intensity for each position of each genotype.

For quantification of Ptch1 FISH, adjacent squares of $20 \mu \mathrm{m}$ side were drawn in the diencephalon, from posterior to anterior, at two apico-basal levels: along the ventricular surface and $\sim 40 \mu \mathrm{m}$ away from the ventricular surface. Total fluorescence intensity was measured in each square on three distinct optical sections. Images from four controls, four $\mathrm{Ftm}^{-/-}$, and three $\left[\mathrm{Ftm}^{-/-}, \mathrm{Gli}^{\Delta /+}\right]$ embryos were used for quantification. For comparison, the measurements were aligned using as a reference the square corresponding to the AP level of the ZLI.

Scanning electron microscopy. Embryos were dissected in $1.22 \times$ PBS, $\mathrm{pH} 7.4,0.1 \mathrm{~m}$ sodium cacodylate, and fixed overnight with $2 \%$ glutaraldehyde in $0.61 \times \mathrm{PBS}, \mathrm{pH} 7.4,0.1 \mathrm{~m}$ sodium cacodylate at $4^{\circ} \mathrm{C}$. Heads were then sectioned to separate the left and right sides of the forebrain, exposing their ventricular surfaces. Head samples were washed several times in $1.22 \times$ PBS and postfixed for $15 \mathrm{~min}$ in $1.22 \times$ PBS containing $1 \% \mathrm{OsO}_{4}$. Fixed samples were washed several times in ultrapure water, dehydrated with a graded series of ethanol, and critical point dried (CPD 300 , Leica) at 79 bar and $38^{\circ} \mathrm{C}$ with liquid $\mathrm{CO}_{2}$ as the transition fluid and then depressurized slowly ( $0.025 \mathrm{bar} / \mathrm{s})$. They were then mounted on aluminum mounts with conductive silver cement. Samples surfaces were coated with a $5 \mathrm{~nm}$ platinum layer using a sputtering device (ACE 600, Leica). Samples were observed under high vacuum conditions using a Field Emission Scanning Electron Microscope (Gemini 500, Zeiss) operating at $3 \mathrm{kV}$, with a $20 \mu \mathrm{m}$ objective aperture diameter and a working distance $\sim 3 \mathrm{~mm}$. Secondary electrons were collected with an in-lens detector. Scan speed and line compensation integrations were adjusted during observation.

Experimental design and statistical analysis. In all experiments, the number of embryos or fetuses analyzed was $\geq 3$ for each genotype, unless otherwise stated. For the comparison of the number of cilia in the different diencephalic regions of control and $\mathrm{Ftm}^{-1-}$ embryos in Figure 10P, quantification was made in four control and four mutant embryos. The number of sections (for cilia density) and of cilia (for cilium length) analyzed are indicated on the graph. In Figure 7, for the quantification of Shh and GFP fluorescence intensity (Fig. 7P,Q), we compared three control, three $\mathrm{Ftm}^{-1-}$, and two $\left[\mathrm{Ftm}^{-1-}, \mathrm{Gli}^{\Delta /+}\right]$ embryos; for Ptch1 intensity (Fig. $7 R, S$ ), we compared four control, four Ftm ${ }^{-1-}$, and three $\left[\mathrm{Ftm}^{-1-}, \mathrm{Gli3}^{\mathrm{\Delta /+}}\right]$ embryos. Statistical analysis was performed using the Prism software. For cilia length (Fig. 10P, bottom graph), unpaired $t$ test was performed. For cilia density (Fig. 10P, top graph) and for Figure $7 P-S$, we used a nonparametric tests (Figs. 10P, top, Mann-Whitney, $7 P-S$, Kruskal-Wallis) because the number of samples was too low to achieve normality of distribution. For Figure $7 P-S$, exact $p$ values are available on request. For quantification of ZLI width, a Mann-Whitney test was used.

\section{Results \\ $\mathrm{Ftm}^{-1-}$ fetuses at the end of gestation display microphthalmia and profound perturbations of the diencephalon and hypothalamus}

Histological analysis combined with dye labeling and immunostaining of axonal tracts showed profound defects in the diencephalon and hypothalamus of $\mathrm{Ftm}^{-1-}$ fetuses at the end of gestation (E18.5; Fig. 1). The ventral regions of the diencephalon and hypothalamus were particularly affected, with a highly dysmorphic ventral part and a perturbed position and shape of the third ventricle (Fig. $1 A-D$ ). In wild-type fetuses, habenular and thalamic nuclei were clearly visible in the dorsal region (Fig. $1 \mathrm{~A}, \mathrm{C})$. In $\mathrm{Ftm}^{-1-}$ fetuses, these nuclei were also present even if their organization was mildly perturbed (Fig. $1 B, D$ ). In contrast, the ventral brain appeared highly disorganized in $\mathrm{Ftm}^{-1-}$ fetuses (Fig. 1A-D). The ventral midline, normally thin in WT, was enlarged in $\mathrm{Ftm}^{-1-}$, likely because of the absence of the most ventral region and fusion of the lateral parts. The most medial hypothalamic nuclei (such as the anteroventral nuclei) were in- distinguishable. The dorsal diencephalon and hypothalamus were present although malformed. In both regions, the axonal tracts [internal capsule (IC) and retroflexus tract (RT)] were disorganized in $\mathrm{Ftm}^{-1-}$ brains (Fig. $1 \mathrm{~B}, \mathrm{D}$, arrowheads). Defects in corticothalamic (CTA) and thalamocortical (TCA) axonal tracts were confirmed with carbocyanine dye labeling (DiI and DiA, respectively) (Fig. $1 E-F^{\prime}$ ). In WT brains, both CTA (magenta) and TCA (green) axons were visualized and colocalized in the IC (Fig. $1 E, E^{\prime}$ ). In $\mathrm{Ftm}^{-1-}$ brains, neither CTA nor TCA grew sufficiently to reach the IC (Fig. $1 F, F^{\prime}$ ). The disorganization of the TCA tracts was confirmed using neurofilament staining (Fig. $1 G, H$ ). In the E18.5 control brain (Fig. $1 G$ ), CTA axons met TCA axons in the IC. In the mutant, thalamic axons were mainly oriented ventrally and the IC was not detected (Fig. $1 H$ ). These axonal defects were confirmed at earlier developmental stages using Neurofilament staining and with Robo3 and Tag1 to label the retroflexus tract (Fig. $1 I-L^{\prime}$ ). The eyes were absent in all $\mathrm{Ftm}^{-/-}$fetuses (Fig. $1 \mathrm{M}-\mathrm{N}^{\prime}$ ), only remnants of the retinal pigmented epithelium were observed under the brain (Fig. $1 N^{\prime}$, arrowhead). We next focused on the developmental origin of these defects.

\section{Patterning of the diencephalon and hypothalamus is affected in $\mathrm{Ftm}^{-1-}$ embryos}

The developing diencephalon is subdivided along the DV axis in roof, alar, basal, and floor plates, and along the caudo-rostral axis in three regions or prosomeres, p1, p2, and p3. The alar plates of $\mathrm{p} 1, \mathrm{p} 2$, and $\mathrm{p} 3$ give rise to the pretectum (PT), thalamus $(\mathrm{TH})$, and prethalamus (PTH), respectively (Fig. 2A). The ZLI is located at the junction between the TH and PTH. The ZLI acts as an organizer for the $\mathrm{TH}$ and $\mathrm{PTH}$, regulating proliferation and cell fate in these two regions (Epstein, 2012; Hagemann and Scholpp, 2012; Zhang and Alvarez-Bolado, 2016).

To investigate diencephalon patterning in Ftm mutants, we performed ISH for genes expressed in these different regions, on coronal and sagittal sections of E13.5 embryos. We first used the alar plate-expressed genes Pax6 (PTH), Gbx2 (TH), and Gad67 (PTH and PT; Fig. 2) encoding, respectively, two transcription factors involved at multiple steps of brain patterning and neurogenesis and a subunit of the glutamate decarboxylase involved in the synthesis of GABA (Stoykova and Gruss, 1994; Stoykova et al., 1996; Miyashita-Lin et al., 1999; Katarova et al., 2000; Hevner et al., 2002). We found that the expression domains of these genes were expanded along the DV axis in $\mathrm{Ftm}^{-1-}$ embryos (Fig. 2). In control embryos, robust Pax6 expression was detected in both the ventricular (VZ) and subventricular zone of the PTH as well as in differentiating neuronal populations (Fig. $2 B, D, F, H$ ). Pax6 was also more faintly expressed in the $\mathrm{VZ}$ of the adjacent regions. In $\mathrm{Ftm}^{-1-}$ embryos, we observed a ventral expansion of the Pax6 expression domain, which now reached the ventral midline (Fig. $2 C, E, G, I$, green arrowheads). In addition, in anterior coronal sections, the hypothalamic, Pax6-negative region was absent from the sections shown (Fig. 2C). Gbx2 expression in control embryos was observed in differentiating neurons of the TH but not in the tegmental areas (TAs) of the diencephalon (Fig. $2 J, L, N, P)$. In $\mathrm{Ftm}^{-1-}$ embryos, Gbx2 expression expanded ventrally (Fig. $2 \mathrm{~K}, \mathrm{M}, \mathrm{O}, \mathrm{Q}$ ). Gad67 expression in the control diencephalon was widespread in neurons of the PT and PTH and absent from diencephalic TA (Fig. 2 R, T,X). In Ftm ${ }^{-1-}$ embryos, the PT and PTH expression domains expanded ventrally (Fig. $2 S, U, Y)$. The ventral expansion of the diencephalic alar plate and the reduction of the basal plate in $\mathrm{Ftm}^{-1-}$ embryos were confirmed using additional marker genes, Ebf1 for PT, $L h \times 2$ for TH 
and Six3 for PTH (Garel et al., 1997; Nakagawa and O'Leary, 2001; Puelles et al., 2006; Fig. 3B-E and data not shown).

The hypothalamus can be subdivided into three main regions, the mammillary area (MAM), the tuberal hypothalamus (TUB) and the anterior hypothalamus (ANT). According to the revised prosomeric model (Puelles et al., 2012; Zhang and Alvarez-Bolado, 2016), the MAM and TUB are in the basal plate of the hypothalamus, while the ANT (also called alar hypothalamus) is in the alar plate (Fig. $3 A$ ). The preoptic area (POA), formerly considered as a hypothalamic region, is actually part of the telencephalon. $N k x 2.1$ is expressed in the hypothalamus in response to Shh signals from the underlying mesendoderm (Dale et al., 1997; Zhao et al., 2012; Blaess et al., 2015). Nkx2.1 is also expressed in two telencephalic structures, the POA and medial ganglionic eminence (MGE; Fig. $3 \mathrm{~F}, \mathrm{H}, \mathrm{J}$ ). In $\mathrm{Ftm}^{-1-}$ embryos, the $N k x 2.1$ expression domain was preserved in the telencephalon (Fig. $3 F, G, J, K)$ but strongly reduced in the hypothalamus (Fig. $3 H-K$ ). Dbx1 expression in progenitors of the TUB (Fig. $3 L, M, P, Q$ ) and MAM (Fig. 3N-Q) regions was severely reduced as well, whereas it was maintained and even expanded in the thalamus (Fig. $3 L-Q$ ). Analysis of Pitx2 (Fig. 3R,S) and Wnt8b (Fig. 3T,U) expression confirmed the reduction in the surface of the MAM in $\mathrm{Ftm}^{-1-}$ embryos. Ebf1 expression in the ANT was still present but fused at the midline (Fig. 3B-E).

These data strongly suggest a severe reduction or loss of the basal plate and ventral midline of the forebrain in $\mathrm{Ftm}^{-1-}$ embryos. Conversely, the alar plate of the diencephalon appears expanded ventrally at all anteroposterior levels.

\section{The rostral thalamus is absent in Ftm $^{-1-}$ embryos}

We took advantage of the expression of two proneural genes, Ngn2, and Mash1/ Ascl1, expressed in distinct and complementary progenitor domains (Fode et al., 2000), to analyze diencephalic subdivisions with greater precision. Ngn2 is expressed in progenitors of most of the $\mathrm{TH}$, in the ZLI and in the TAs of the diencephalon, in a domain in the POA and in the dorsal telencephalon (Fode et al., 2000; Vue et al., 2007; Fig. 4B, D, F, H). Mash1 is expressed in progenitors of the PTH, in the prospective rostral thalamus (TH-R; see next paragraph) and in different hypothalamic subdivisions (McNay et al., 2006; Vue et al., 2007; Kim et al., 2008; Fig. 4 J, L, N, P). In Ftm ${ }^{-1-}$ embryos, $\mathrm{Ngn} 2$ expression was lost in the TA (empty arrowheads) and $\mathrm{mm}$; (in $\boldsymbol{V}) \boldsymbol{V}, \boldsymbol{W}, 100 \mu \mathrm{m}$.
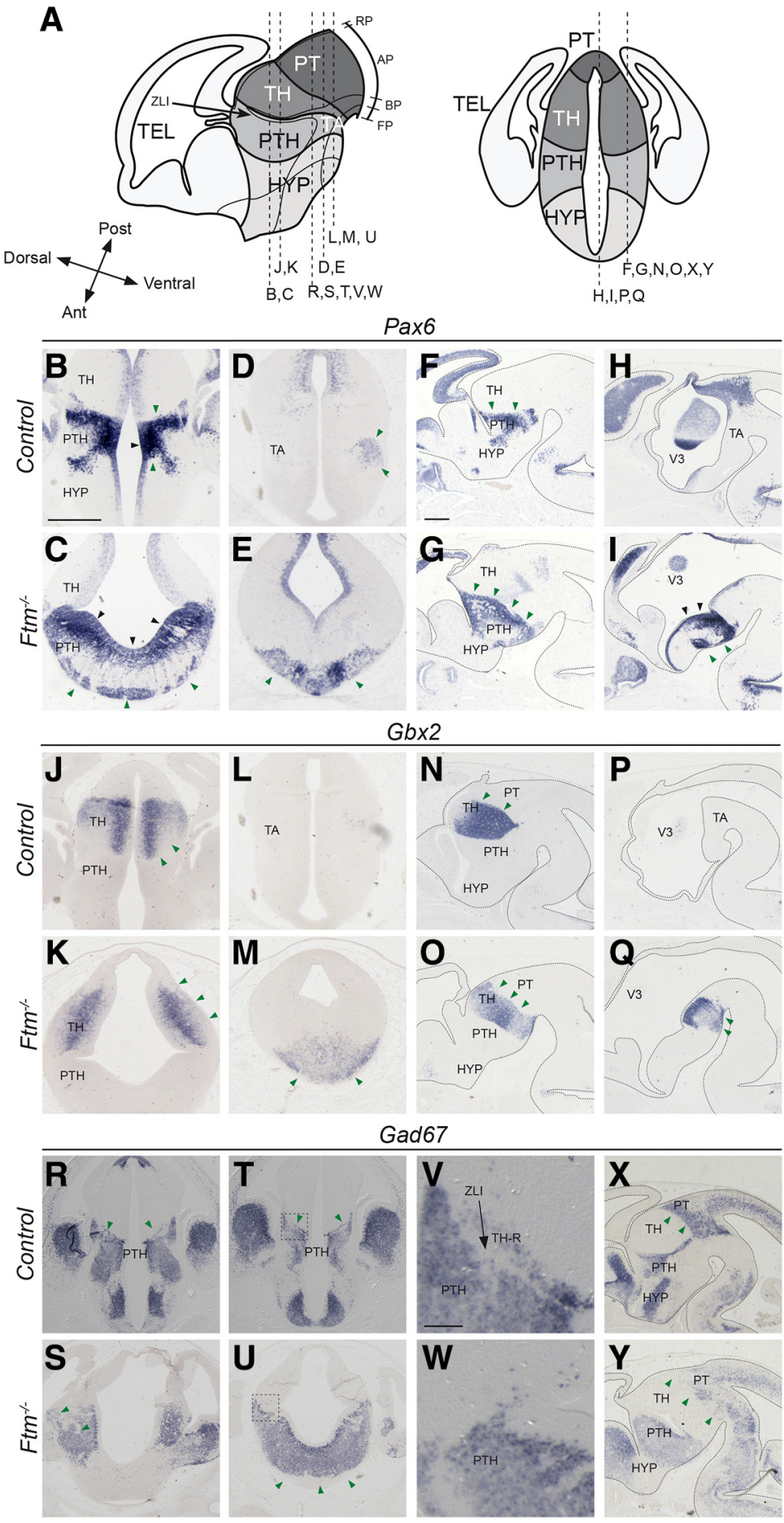

Figure 2. Diencephalon patterning at E13.5. A, Schematic drawings of the E13.5 forebrain in sagittal (left) and coronal (right) views. The position of the coronal $(\boldsymbol{B}-\boldsymbol{E}, \boldsymbol{J}-\boldsymbol{M}, \boldsymbol{R}-\boldsymbol{W})$ and sagittal $(\boldsymbol{F}-\boldsymbol{I}, \boldsymbol{N}-\boldsymbol{Q}, \boldsymbol{X}, \boldsymbol{Y})$ sections shown below is indicated with dashed lines. Note that in the left diagram, anteroposterior and dorsoventral axes are indicated at the level of the $Z L I . B-Y, I S H$ with probes for Pax6 $(\boldsymbol{B}-\boldsymbol{I}), \mathbf{G b \times 2}(\boldsymbol{J}-\boldsymbol{Q})$, and Gad67 $(\boldsymbol{R}-\boldsymbol{Y})$ in coronal sections at two distinct anteroposterior levels $(\boldsymbol{B}-\boldsymbol{E}, \boldsymbol{J}-\boldsymbol{M}, \boldsymbol{R}-\boldsymbol{W})$ and in sagittal sections at lateral $(\boldsymbol{F}, \mathbf{G}, \mathbf{N}, \mathbf{O}, \boldsymbol{X}, \boldsymbol{Y})$ and medial $(\boldsymbol{H}, \boldsymbol{I}, \boldsymbol{P}, \mathbf{Q})$ levels. The genotype (control or $F \mathrm{Fm}^{-1-}$ ) is indicated on the left, where control stands for $\mathrm{Ftm}^{+/+}$or $\mathrm{Ftm}^{+/-}$. In sagittal sections, the brain is outlined with dotted lines. Black and green arrowheads point to neuronal progenitors and neurons, respectively. Ant, Anterior; BP, basal plate; $\mathrm{FP}$, floor plate; RP, roof plate; Post, posterior; TEL, telencephalon; $3 \mathrm{~V}$, third ventricle. Scale bars: (in $\boldsymbol{B}$ for coronal sections, in $\boldsymbol{F}$ for sagittal sections), $\boldsymbol{A}-\boldsymbol{U}, \boldsymbol{X}, \boldsymbol{Y}, 0.5$

activated ectopically in a salt-and-pepper manner in regions adjacent to the telencephalon (black arrowheads), suggesting a perturbation of the telencephalic-diencephalic boundary (Fig. $4 C, E, G, I)$. Mash 1 was still expressed in the PTH and hypothala- 
A
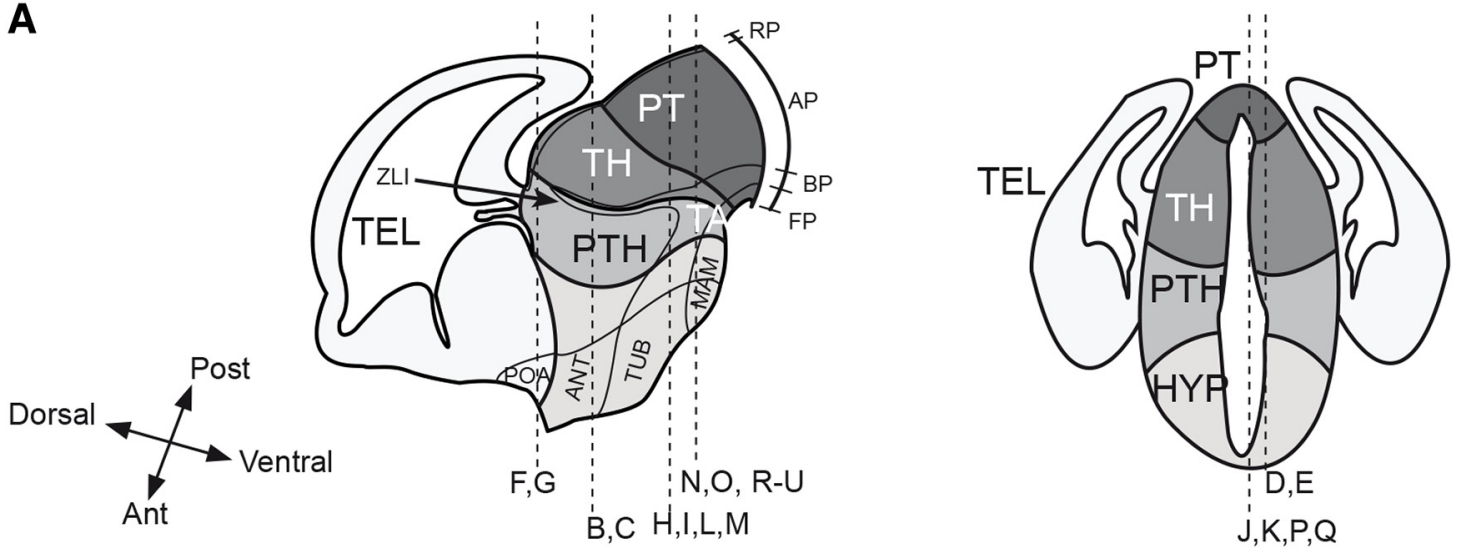

Ebf1

$N k \times 2.1$
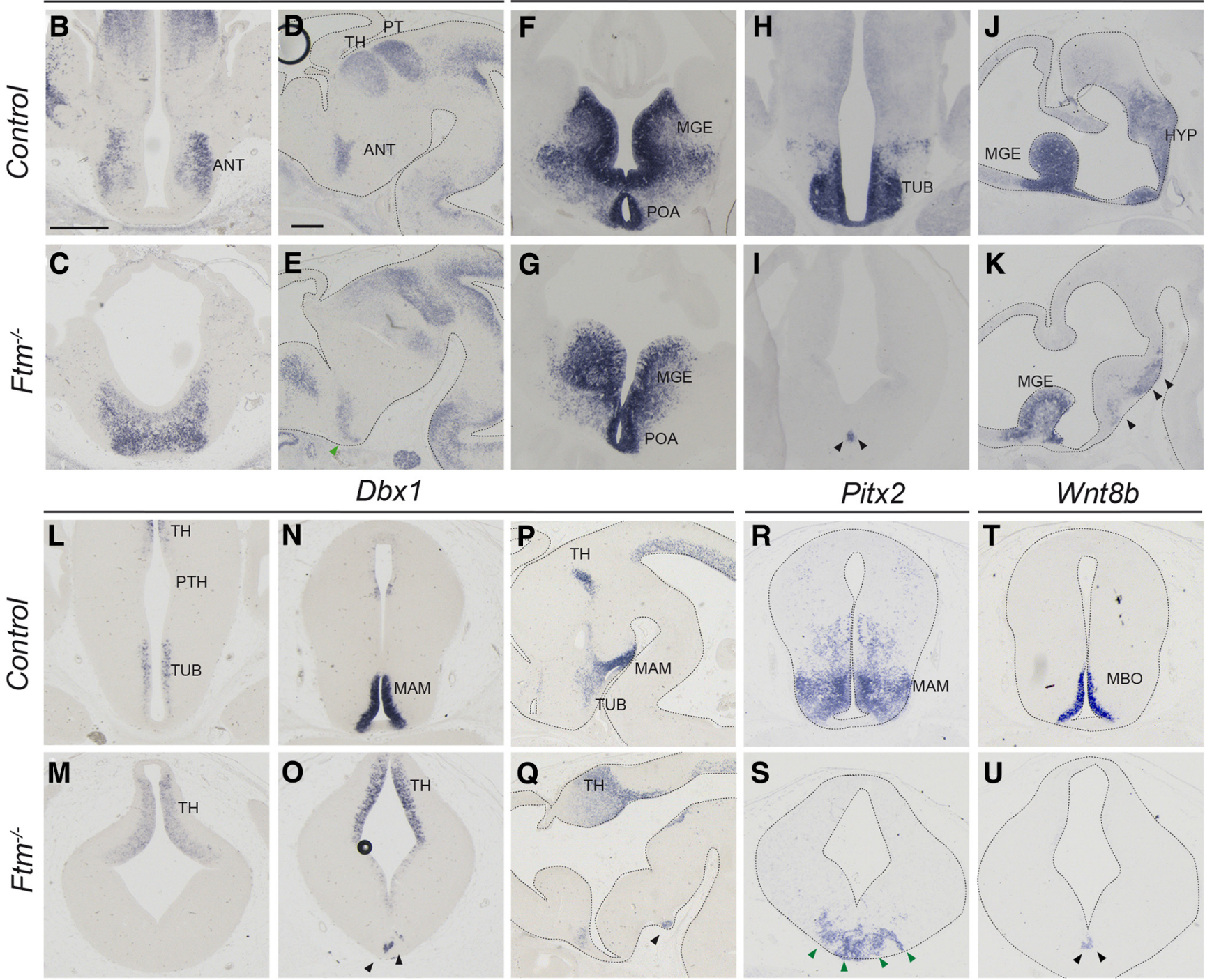

Figure 3. Hypothalamus patterning at E13.5. $\boldsymbol{A}$, Schematic drawings of the E13.5 forebrain in sagittal (left) and coronal (right) views. The position of the coronal $(\boldsymbol{B}, \boldsymbol{C}, \boldsymbol{F}-\boldsymbol{I}, \boldsymbol{L}-\mathbf{O}, \boldsymbol{R}-\boldsymbol{U})$ and sagittal $(\boldsymbol{D}, \boldsymbol{E}, \boldsymbol{J}, \boldsymbol{K}, \boldsymbol{P}, \boldsymbol{Q})$ sections shown below is indicated with dashed lines. Note that in the left diagram, anteroposterior and dorsoventral axes are indicated at the level of the hypothalamus. $\boldsymbol{B}-\boldsymbol{U}, \mathbf{I S H}$

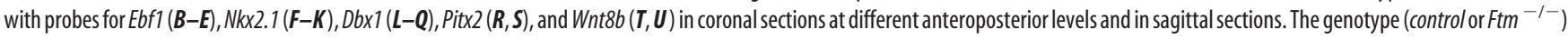
is indicated on the left. Black and green arrowheads point to neuronal progenitors and neurons, respectively. In sagittal sections and in coronal sections in $\boldsymbol{R}-\boldsymbol{U}$, the brain is outlined with dotted lines. Ant, Anterior; AP, alar plate; BP, basal plate; $F$, floor plate; $M B O$, mammillary body; Post, posterior; RP, roof plate; TEL, telencephalon. Scale bars: (in $\boldsymbol{B}$ for coronal sections, in D for sagittal sections), $0.5 \mathrm{~mm}$. 
mus (HYP; Fig. $4 K, M, O, Q$ ), but very reduced caudally (in the MAM; Fig. 4Q, black arrowheads). The analysis of $\mathrm{Ngn} 2$ and Mash1 expression also revealed a thickening of the progenitor domains in the TH and PTH at E12.5-E13.5 (Fig. 4B$Q)$, suggesting a delay in neurogenesis and/or an increased proliferation potential of forebrain progenitors in $\mathrm{Ftm}^{-1-}$ embryos.

The nested domains of $\mathrm{Ngn} 2$ and Mash1 expression in the diencephalon prefigure the intrinsic subdivision of the thalamus into anterior (TH-R) and posterior [caudal thalamus (TH-C)] territories (Vue et al., 2007; Fig. 4B,J). Ngn2 and Mash1 domains in the $\mathrm{TH}$ and $\mathrm{PTH}$, respectively, were continuous in $\mathrm{Ftm}^{-1-}$ embryos, suggesting a perturbation of thalamic subdivisions (Fig. $4 C, K$, asterisks). This was confirmed by a closer examination of Ngn2 and Mash1 nested expression domains (Fig. $4 R-A A$ ). We performed combined Shh/Ngn2 (Fig. $4 V, W$ ), Shh/Mash1 (Fig. $4 X, Y$ ) and Ngn2/Mash1 (Fig. 4Z,AA) fluorescence ISH and immunostaining to analyze the relationship of the different diencephalic domains with respect to the ZLI. In $\mathrm{Ftm}^{-1-}$ embryos, the domain of Mash1 expression posterior to the Shh-positive ZLI was lost (Fig. 4Y, white asterisks). The Ngn2-positive TH-C and Mash1positive PTH domains abutted at the level of the ZLI (Fig. 4W,Y,AA).

The TH-R contributes to GABAergic nuclei that participate in the subcortical visual shell, involved in the entrainment of the circadian rhythm (Delogu et al., 2012). Thus, neurons of the TH-R express Gad67 like those of the PTH, while neurons of the TH-C do not (Fig. $2 \mathrm{~V}$ ). In $\mathrm{Ftm}^{-1-}$ embryos, the stripe of Gad67 expression in the thalamus was absent, confirming the loss of the TH-R (Fig. $2 V, W)$.

In conclusion, the $\mathrm{TH}-\mathrm{R}$ is lost in $\mathrm{Ftm}^{-1-}$ embryos and the TH-C now abuts the ZLI. A diagram summarizing the Ftm mutant forebrain phenotype is provided in Figure 11A.

Optic vesicles form in $\mathrm{Ftm}^{-/-}$embryos and display patterning defects

Because eyes were absent in $\mathrm{Ftm}^{-1-} \mathrm{fe}-$ tuses at the end of gestation (Fig. $1 I-L$ ), we investigated eye formation and patterning at E11.5. Eye development begins with the formation of the eye field in the alar hypothalamus and its separation into two bilaterally symmetrical optic vesicles. The expanding optic vesicles induce the surface ectoderm to form the lens placodes.
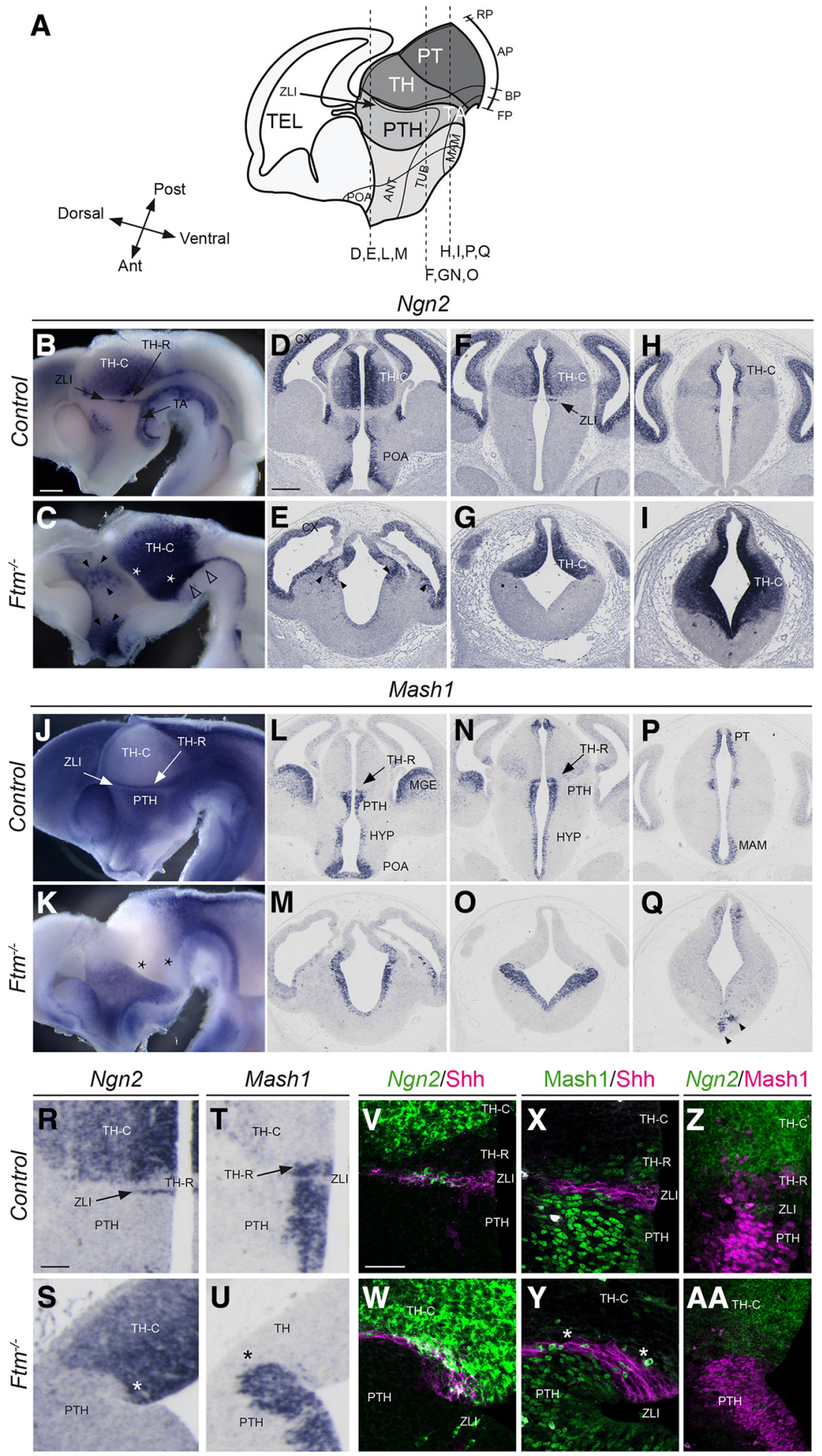

Figure 4. Progenitor domains at E12.5-E13.5. $A$, Schematic drawings of the E13.5 forebrain in sagittal view. The position of the coronal sections $(\boldsymbol{D}-\boldsymbol{I}, \boldsymbol{L}-\mathbf{Q})$ shown below is indicated with dashed lines. $\boldsymbol{B}-\boldsymbol{U}, \mathbf{I S H}$ with probes for $\operatorname{Ngn} 2(\boldsymbol{B}-\boldsymbol{I}, \boldsymbol{R}, \boldsymbol{S})$ and Mash1 $(\boldsymbol{J}-\boldsymbol{Q}, \boldsymbol{T}, \boldsymbol{U})$ in whole-mount hybridization on sagittally-bisected brains viewed from the ventricular side $(\boldsymbol{B}, \boldsymbol{C}, \boldsymbol{J}, \boldsymbol{K})$ or on coronal sections at different anteroposterior levels $(\boldsymbol{D}-\boldsymbol{I}, \boldsymbol{L}-\boldsymbol{Q}, \boldsymbol{R}-\boldsymbol{U})$. The genotype is indicated on the left. $\boldsymbol{V}, \boldsymbol{W}$, Shh immunofluorescence (magenta) combined with Ngn2 fluorescence ISH (green). $\boldsymbol{X}, \boldsymbol{Y}$, Double-immunofluorescence for Shh (magenta) and Mash1 (green). $Z, A A$, Mash immunofluorescence (magenta) combined with Ngn2 fluorescence ISH (green). C, E, Black arrowheads point to patchy Ngn2 expression in the prethalamus and white arrowheads point to missing Ngn2 expression domain in the ventral forebrain. $\mathbf{Q}$, Black arrowheads point to remnants of the MAM. $\boldsymbol{C}, \boldsymbol{K}, \boldsymbol{S}, \boldsymbol{Y}$, Asterisks point to the absence of the Ngn2-negative, Mash1-positive TH-R in Ftm ${ }^{-1-}$ embryos. Ant, anterior; AP, alar plate; BP, basal plate; FP, floor plate; RP, roof plate; Post, posterior; TEL, telencephalon. Scale bars: (in $\boldsymbol{B}$ for whole-mount ISH, in $\boldsymbol{D}$ for coronal sections) $\boldsymbol{B}-\boldsymbol{Q}, 0.5 \mathrm{~mm}$; (in $\boldsymbol{R}) \boldsymbol{R}-\boldsymbol{U}, 100 \mu \mathrm{m}$; (in $\boldsymbol{V}$ ) $\boldsymbol{V}-\mathbf{A} A, 50 \mu \mathrm{m}$. 

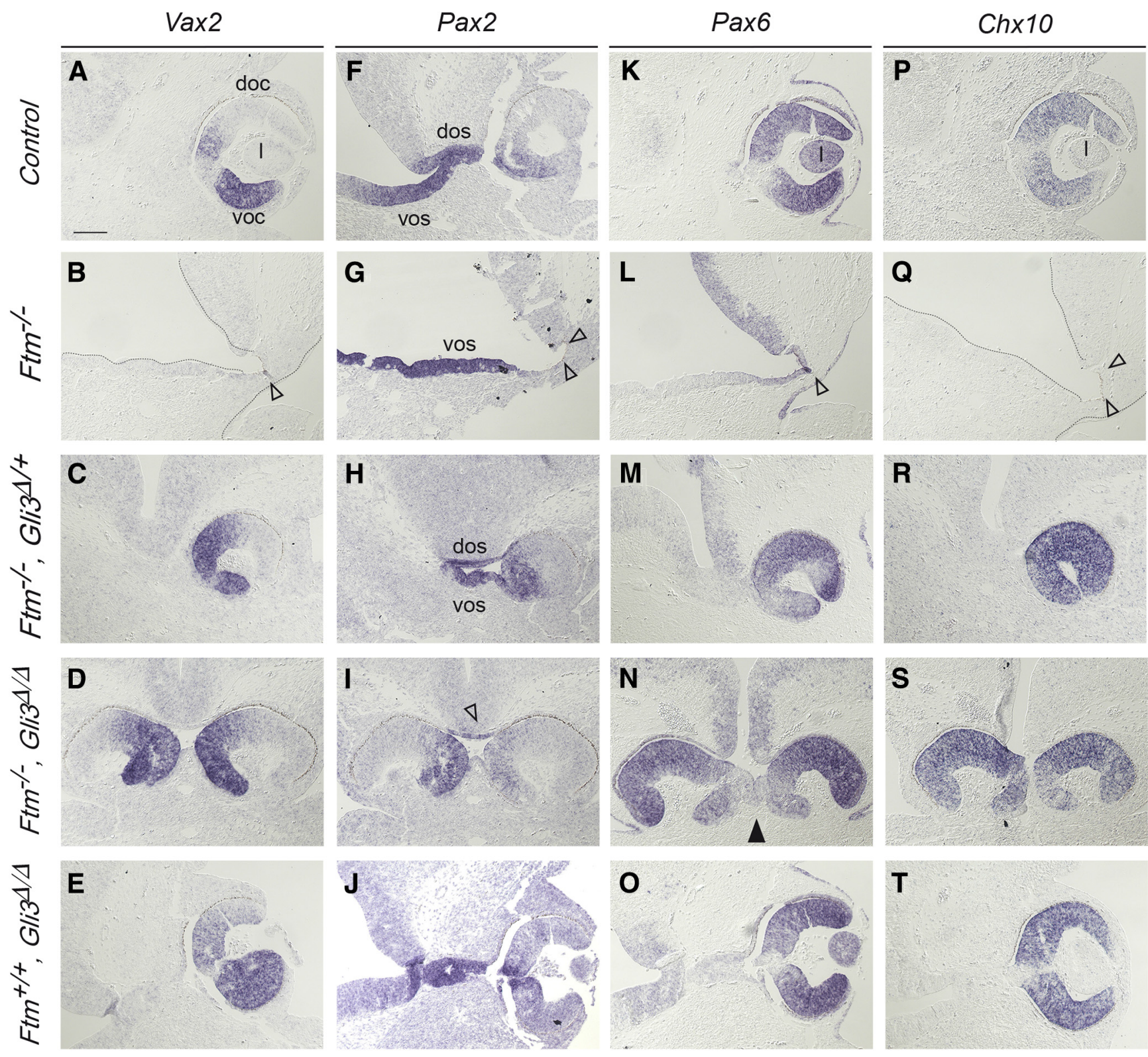

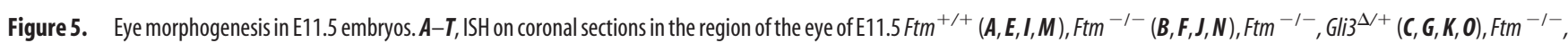
$G_{l i 3}{ }^{\Delta / \Delta}(\boldsymbol{D}, \boldsymbol{H}, \boldsymbol{L}, \boldsymbol{P})$, and $F t m^{+/+}, G l i 3^{\Delta / \Delta}(\boldsymbol{E}, \boldsymbol{J}, \boldsymbol{O}, \boldsymbol{T})$ with probes for $\operatorname{Vax} 2(\boldsymbol{A}-\boldsymbol{E}), \operatorname{Pax2}(\boldsymbol{F}-\boldsymbol{J}), \operatorname{Pax6}(\boldsymbol{K}-\mathbf{O})$, and $C h x 10(\boldsymbol{P}-\boldsymbol{T}) . \boldsymbol{B}, \boldsymbol{G}, \boldsymbol{L}, \boldsymbol{Q}$, Empty arrowheads point to the missing optic cup; empty arrowhead in I points to the reduced optic stalk. $N$, Black arrowhead points to partially fused optic cups. doc, Dorsal optic cup; dos, dorsal optic stalk; I, lens; voc, ventral optic cup; vos, ventral optic stalk. Scale bars: (in $A) 100 \mu \mathrm{m}$.

The optic vesicle separates into the optic stalk proximally and the optic cup distally. Then the optic cup invaginates with the lens placode, forming two layers, the outer layer differentiates into the retinal pigmented epithelium (RPE) and the inner layer into the neural retina (Furimsky and Wallace, 2006).

We analyzed the expression patterns of the Pax2, Vax2, Pax6, and $C h \times 10$ transcription factor genes, which define distinct eye territories (Furimsky and Wallace, 2006). At this stage, Pax6 and Pax2 are expressed in the optic cup and optic stalk, respectively (Fig. $5 F, K$ ), where they repress each other. Pax6 is required for optic cup formation, whereas Pax2-null mice display increased optic cups at the expense of optic stalk (Schwarz et al., 2000). Chx10 is also expressed in the optic cup (Fig. 5P). Vax2 is expressed in the ventral domain of the optic cup (Fig. 5A), where it promotes ventral optic fates. In $\mathrm{Ftm}^{-1-}$ embryos, the neural retina was absent as assessed by the absence of Chx10 and Vax2 expression (Fig. 5B,Q). Only a tiny region of the RPE could be detected thanks to cell pigmentation (Fig. $5 B, G, L$, empty arrowheads). Pax2 was expressed, indicating the presence of the optic stalk (Fig. 5G), which suggests correct eye-field separation. Consistently, optic vesicles formed in $\mathrm{E} 9 \mathrm{Ftm}^{-1-}$ embryos as in controls (Fig. 6S,U,V).

In conclusion, in $\mathrm{Ftm}^{-1-}$ embryos, eye-field separation occurs correctly but proximodistal patterning of the optic vesicle is incorrect, leading to an absence of the optic cup and of lens induction.

\section{Shh expression and pathway activity are impaired in the forebrain of $\mathrm{Ftm}^{-/-}$embryos}

The reduction of the ventral forebrain in Ftm mutants suggests defects in the Hh pathway. To test this hypothesis, we analyzed Shh signaling activity in the forebrain by ISH and IF for Shh itself 

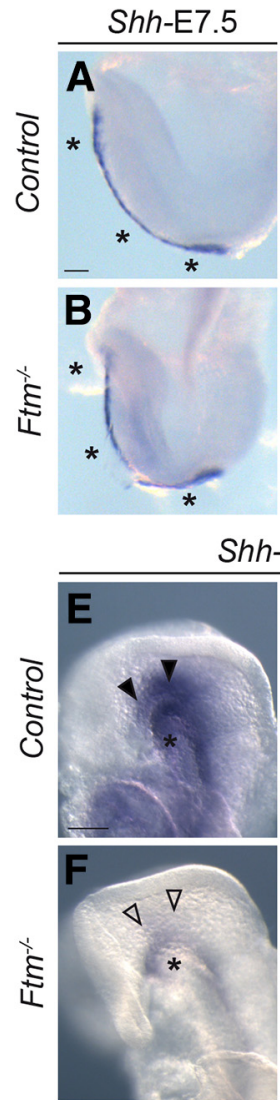

Shh-E8.5

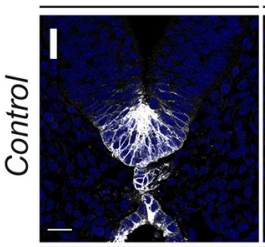

FoxA2-E8.5
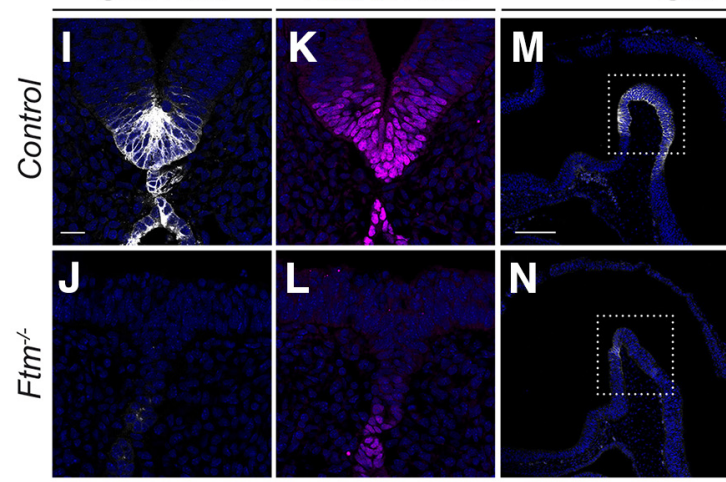

Shh/GFP-E9.0
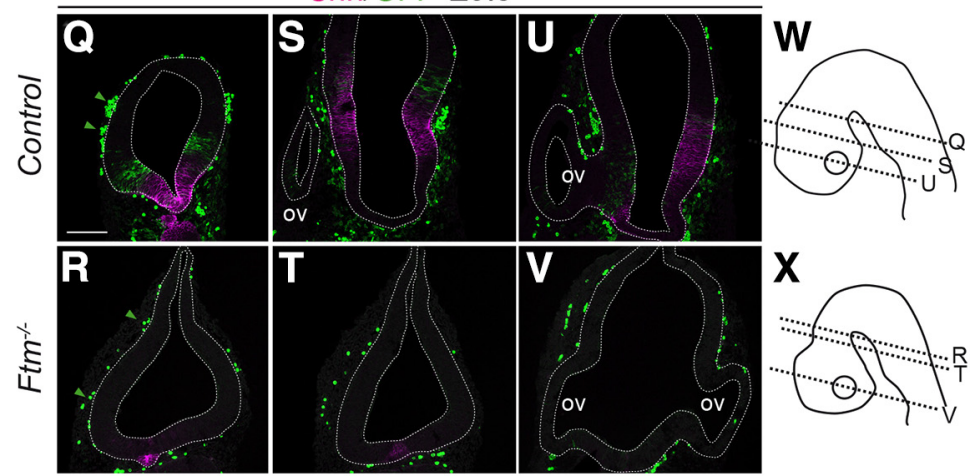

Figure 6. Shh expression and signaling in the E7.5-E9.5 embryo forebrain. $\boldsymbol{A}-\boldsymbol{H}$, Whole-mount ISH on $\mathrm{E} 7.5(\boldsymbol{A}, \boldsymbol{B}), \mathrm{E} 8.0\left(\boldsymbol{C}-\boldsymbol{D}^{\prime \prime}\right)$, and E8.5 $\left(\boldsymbol{E}-\boldsymbol{H}^{\prime}\right)$ embryos with probes for $\operatorname{Shh}\left(\boldsymbol{A}, \boldsymbol{B}, \boldsymbol{E}-\boldsymbol{F}^{\prime}\right)$ or Ptch $1\left(\boldsymbol{C}-\boldsymbol{D}^{\prime \prime}, \mathbf{G}-\boldsymbol{H}^{\prime \prime}\right) . \boldsymbol{C}-\boldsymbol{D}^{\prime \prime}$, Lateral, ventral, and top views of the anterior neural plate of the same control and $F m^{-/-}$embryos, respectively. $\boldsymbol{E}^{\prime}, \boldsymbol{F}^{\prime}$, Vibratome sections at the level of the forebrain of embryos seen in $\boldsymbol{E}$ and $\boldsymbol{F}$, respectively. $\boldsymbol{G}-\boldsymbol{H}^{\prime}$, Lateral and ventral views of the anterior neural plate of the same control and $\mathrm{Ftm}^{-1-}$ embryos, respectively. Black arrowheads indicate Shh and Ptch1 expression sites in the neural plate; black asterisks indicate Shh expression in the mesoderm underlying the neural plate. $\boldsymbol{D}, \boldsymbol{F}, \boldsymbol{H}$, Empty arrowheads indicate severe reduction of $\mathrm{Sh}$ and Ptch1 expression in the neural plate of $\mathrm{Ftm}^{-1-}$ embryos. Red arrowheads point to examples of cells with remnants of $\mathrm{Shh}$ and for the Hh target genes Ptc1, Gli1, and FoxA2. In addition, to obtain a contextindependent assay of $\mathrm{Hh}$ transcriptional activity through Gli transcription factors binding to their DNA targets, we introduced into the Ftm mutant background the $\mathrm{Tg}[\mathrm{GBS}:$ :GFP] reporter transgenic line in which GFP expression is driven by a concatemer of Gli-binding sites (Balaskas et al., 2012).

In mouse embryos, Shh is initially expressed from E7.5 in axial tissues underlying the neural plate (notochord posteriorly and prechordal plate anteriorly), where it signals to the overlying neural plate to induce ventral structures. Shh signaling induces Shh expression in the ventral forebrain from E8.0 onward (Dale et al., 1997). Whereas Shh expression in the axial mesoderm was unperturbed in E7.5 $\mathrm{Ftm}^{-1-}$ embryos compared with controls (Fig. 6A, $B$, asterisks), Ptch1 expression in the ventral neural plate (Fig. $6 C-C^{\prime}$, black arrowheads) was reduced in $\mathrm{Ftm}^{-1-}$ (Fig. $6 D-D^{\prime \prime}$, empty arrowheads) with few remaining Ptch1positive cells (Fig. $6 D-D^{\prime \prime}$, red arrowheads). In E8.5 control embryos, Shh is still expressed in the mesendoderm underlying the brain (Fig. $6 E, E^{\prime}$, asterisks, $I$ ). In addition, it is activated in the ventral neural tube, including the ventral forebrain (Dale et al., 1997; Alvarez-Bolado et al., 2012; Fig. 6E, $E^{\prime}$, black arrowheads, $I$ ). In E8.5 $\mathrm{Ftm}^{-1-}$ embryos, Shh expression persisted in the notochord and prechordal plate (Fig. $6 F, F^{\prime}$,J, black asterisks) but was severely downregulated in the ventral neural tube and brain (empty arrowheads) with few remaining positive cells (red arrowhead). Ptch1 expression in two stripes surrounding the Shh expression domain in the ventral neural tube and brain of control embryos (Fig. 6G, $G^{\prime}$, black arrowheads) was also downregulated in $\mathrm{Ftm}^{-1-}$ (Fig. $6 \mathrm{H}, \mathrm{H}^{\prime}$, empty arrowheads), consistent with the reduction of Shh expression. FoxA2, a target of Shh signaling expressed in the ventral floor plate and in the ventral forebrain (Hallo-

or Ptch1 expression. $\boldsymbol{I}-\boldsymbol{P}$, IF for Shh $(\boldsymbol{I}, \boldsymbol{J}, \boldsymbol{M}-\boldsymbol{P})$ and FoxA2 $(\boldsymbol{K}, \boldsymbol{L})$ on coronal sections of $E 8.5(I-L)$ and sagittal sections of $E 9.5$ $(\boldsymbol{M}-\boldsymbol{P})$ embryos.P, White arrowhead points to the small dot of Shh expression in the basal plate of $\mathrm{Ftm}^{-/-}$embryos. Q-V, Double IF for Shh and GFP in Tg[GBS::GFP] transgenic embryos. Green arrowheads point to GFP-positive blood cells. The genotypes are indicated on the left. Control stands for $\mathrm{Ftm}^{+/+}$or $\mathrm{Ftm}^{+/-} . \boldsymbol{W}, \boldsymbol{X}$, Schematics indicating the approximate levels of sections in $\mathbf{Q}-\boldsymbol{V}$. Note that the sections are tilted, so they do not look bilaterally symmetric. $0 v$, Optic vesicle. Scale bars: (in A) $\boldsymbol{A}, \boldsymbol{B}$, (in $\boldsymbol{C}$ ) $\boldsymbol{C}-\boldsymbol{D}^{\prime \prime}$, (in $\left.\boldsymbol{E}\right) \boldsymbol{E}-\boldsymbol{H}^{\prime}, 50 \mu \mathrm{m}$; (in $\left.\boldsymbol{I}\right) \boldsymbol{I} \boldsymbol{L}, 20 \mu \mathrm{m}$; (in $M$ ), $M, N, 500 \mu \mathrm{m}$; (in 0) 0, P, (in Q) $\mathbf{Q}-\boldsymbol{V}, 100 \mu \mathrm{m}$. 

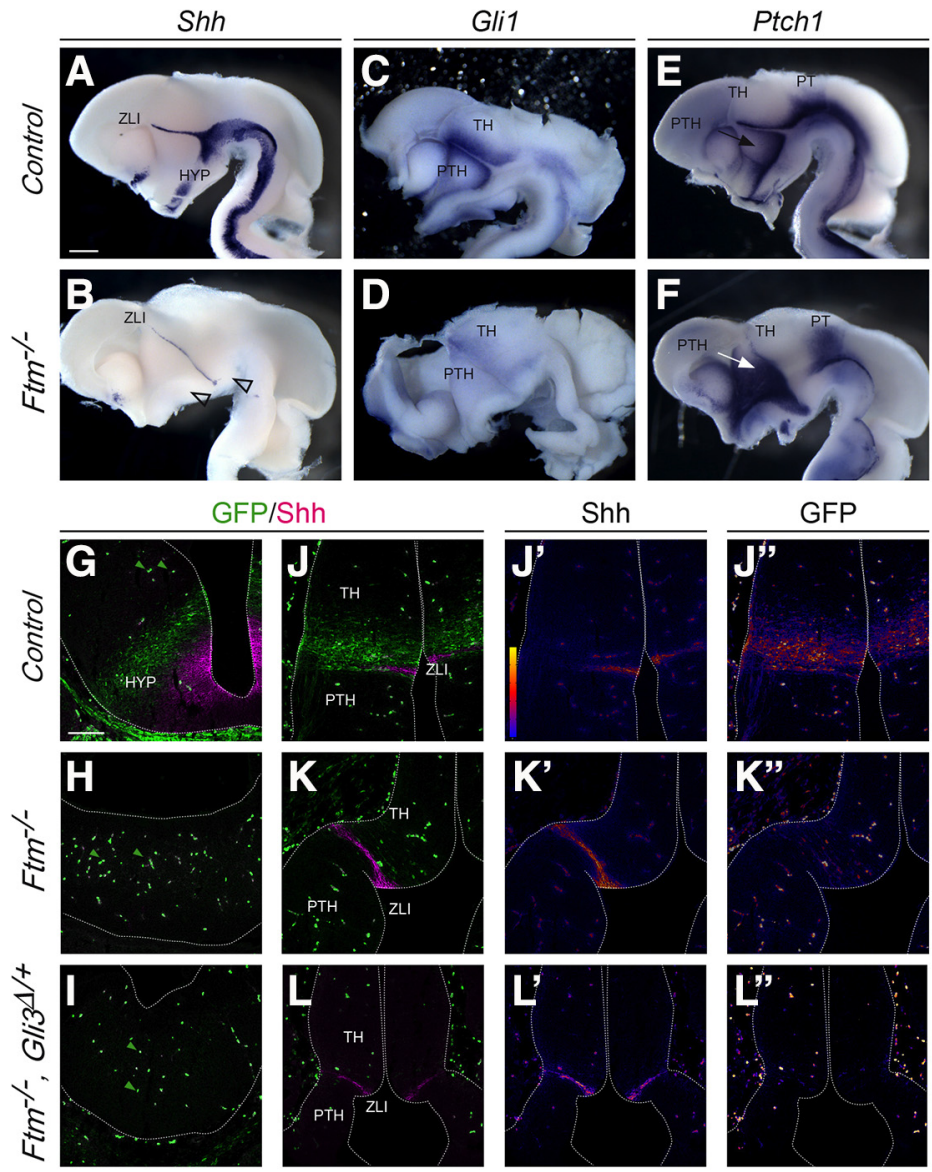
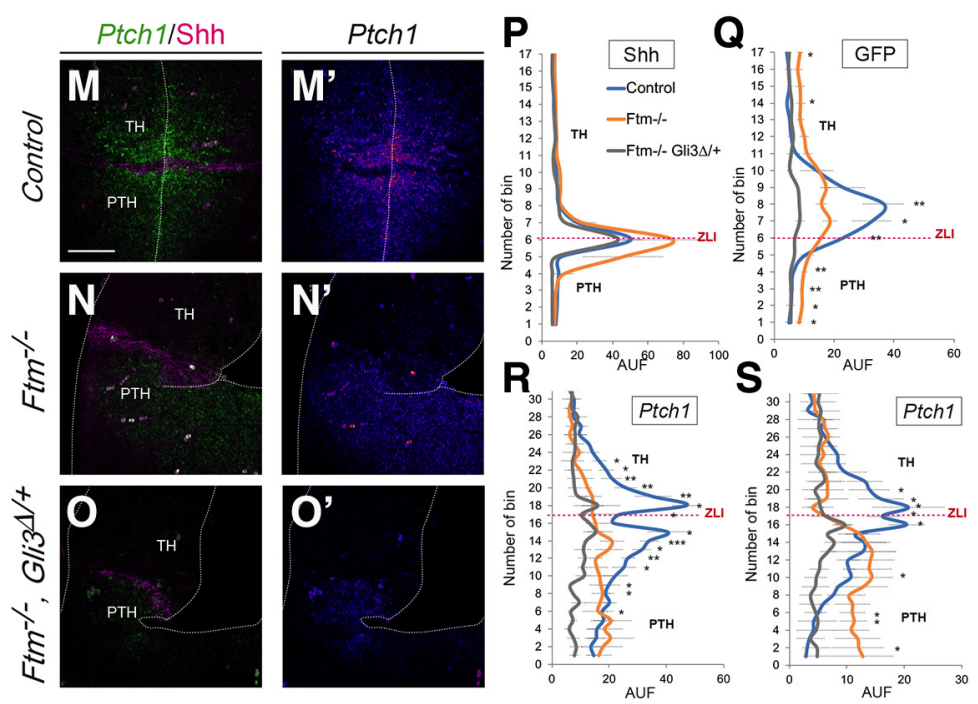

Figure 7. Shh expression and signaling in the E12.5 embryo forebrain. $\boldsymbol{A}-\boldsymbol{F}$, Whole-mount ISH on E12.5 $\operatorname{control}(\boldsymbol{A}, \boldsymbol{C}, \boldsymbol{E})$ or $F t^{-l-}(\boldsymbol{B}, \boldsymbol{D}, \boldsymbol{F})$ half-brains viewed from the ventricular surface, with probes for $\operatorname{Shh}(\boldsymbol{A}, \boldsymbol{B}), \mathrm{Gli1}(\boldsymbol{C}, \boldsymbol{D}), \operatorname{or} \operatorname{Ptch} 1(\boldsymbol{E}, \boldsymbol{F})$. $\boldsymbol{G}-\boldsymbol{L}^{\prime \prime}$, IF on coronal sections of control $\left(\boldsymbol{G}, \boldsymbol{J}, \boldsymbol{J}^{\prime}, \boldsymbol{J}^{\prime \prime}\right), \mathrm{Ftm}^{-\boldsymbol{-}}\left(\boldsymbol{H}, \boldsymbol{K}, \boldsymbol{K}^{\prime}, \boldsymbol{K}^{\prime \prime}\right)$ or $\left[\right.$ Ftm, Gli3 $\left.{ }^{\Delta /+}\right]\left(\boldsymbol{I}, \boldsymbol{L}, \boldsymbol{L}^{\prime}, \boldsymbol{L}^{\prime \prime}\right) \mathrm{Tg}[\mathrm{GBS}:: \mathrm{GFP}]$ embryos. IF was performed with antibodies for Shh and GFP. $\boldsymbol{J}^{\prime}, \boldsymbol{J}^{\prime \prime}, \boldsymbol{K}^{\prime}, \boldsymbol{K}^{\prime \prime}, \boldsymbol{L}^{\prime}, \boldsymbol{L}^{\prime \prime}$, Fire versions of $\operatorname{Sh}$ and GFP are shown $\left(\boldsymbol{J}^{\prime}\right.$ fire scale). $\boldsymbol{B}$, Empty arrowheads point to the missing Shh expression domain in the ventral forebrain. $\mathbf{M}-\mathbf{O}^{\prime}, \mathbf{C}^{\prime}$ Combined fluorescence ISH Ptch1 and IF for Shh on coronal sections for of the diencephalon of E12.5 control $\left(\mathrm{M}, \mathrm{M}^{\prime}\right), \mathrm{Ftm}^{-/-}\left(\boldsymbol{N}, \boldsymbol{N}^{\prime}\right)$ and compound $\left[\mathrm{Ftm}^{-1-}, \mathrm{Gli}^{\Delta /+}\right]\left(\mathbf{0}, \mathbf{0}^{\prime}\right)$ embryos. $\boldsymbol{M}^{\prime}-\mathbf{O}^{\prime}$, Fire versions of Ptch1 FISH. $\boldsymbol{M}-\mathbf{0}$, Green arrowheads point to GFP-positive blood cells. $\boldsymbol{P}$-S, Diagrams showing the quantification of the intensity of $\operatorname{Shh}(\boldsymbol{P})$ or GFP $(\mathbf{Q})$ IF and Ptch1 FISH $(\boldsymbol{R}, \boldsymbol{S})$ along the diencephalon. Ptch1 FISH intensity was quantified next to the ventricular surface $(\boldsymbol{R})$ or $40 \mu \mathrm{m}$ away from the ventricular surface $(\boldsymbol{S})$. Numbers on the abscissa relate to the position of the squares of quantification. Fluorescence intensity in ordinate is given in arbitrary units (AUF). Q-S, P values of statistical tests are shown as ${ }^{*} p<0.1,{ }^{* *} p<0.01$, and ${ }^{* * *} p<0.001$. No asterisk means that the difference was found nonsignificant by the statistical test. Scale bars: (in $\boldsymbol{A}$ ) $\boldsymbol{A}-\boldsymbol{F}, 0.5 \mathrm{~mm}$; (in $\mathbf{G}) \mathbf{G}-\mathbf{O}^{\prime}, 100 \mu \mathrm{m}$. net et al., 2002; Ribes et al., 2010; Fig. 6K), was faintly expressed in the neural plate of $\mathrm{Ftm}^{-1-}$ embryos, confirming the very low $\mathrm{Hh} / \mathrm{Gli}$ activity (Fig. $6 L$ ).

At E9.0-E9.5, Shh expression is still present in the ventral diencephalon (Fig. $6 M, O, Q)$ but in the hypothalamus it is downregulated in the most ventral region and activated in two lateral stripes in the basal plate (Fig. 6S,U; Szabó et al., 2009a; Alvarez-Bolado et al., 2012, Blaess et al., 2015). Shh expression was lost in the whole ventral forebrain of $\mathrm{Ftm}^{-1-}$ embryos, except in a tiny spot in the diencephalic ventral midline located approximately at the anteroposterior (AP) level of the future ZLI (Fig. $6 N, P, R, T, V$ ). Gli activity assessed by Tg[GBS::GFP] was observed lateral to Shh expression domain in all forebrain regions of control embryos (Fig. 6Q,S,U), whereas in $\mathrm{Ftm}^{-1-}$ embryos, Gli activity could not be detected (Fig. $6 R, T, V$ ). Note that GFP is also expressed in blood cells and that this expression was still present in $\mathrm{Ftm}^{-1-}$ embryos (Balaskas et al., 2012; Fig. 6Q,R, green arrowheads).

Overall our results show that Shh signaling activity is drastically reduced but not totally abolished in the ventral forebrain of $\mathrm{Ftm}^{-1-}$ embryos as early as E8.0.

\section{Shh expression and $\mathrm{Hh} / \mathrm{Gli}$ pathway} activity show different perturbations in distinct domains of the E12.5

\section{diencephalon}

We next investigated $\mathrm{Hh} / \mathrm{Gli}$ pathway activity at E12.5, when the ZLI is fully formed and secretes Shh to organize cell fate in the thalamus and prethalamus (Epstein, 2012; Zhang and Alvarez-Bolado, 2016). The ZLI was formed in both control and $\mathrm{Ftm}^{-1-}$ embryos, and its DV extent was increased in $\mathrm{Ftm}^{-1-}$ compared with control embryos (Fig. $7 A, B$ ). In contrast, Shh expression was absent from the ventral forebrain of $\mathrm{Ftm}^{-1-}$ embryos (Fig. 7B, empty arrowheads). To test whether Shh signaling was active at the ZLI, we analyzed Ptch1 and Gli1 expression as well as Gli transcriptional activity with $\mathrm{Tg}[\mathrm{GBS}:$ :GFP]. Surprisingly, Gli1 and Ptch1 were differently affected in $\mathrm{Ftm}^{-1-}$ embryos (Fig. 7C-F). Gli1 expression was dampened in the regions close to the Shh expression domains (Fig. $7 C, D)$. In contrast, Ptch1 expression was totally downregulated in the thalamus and upregulated in the prethalamus and pretectum (Fig. $7 E, F$ ). Using FISH on sections and signal quantification, we confirmed the differential expression of Ptch1 on both sides of the ZLI in $\mathrm{Ftm}^{-/-}$ embryos and found that this upregulation 
was more striking at a distance from the ventricular surface (Fig. $7 M-N^{\prime}, R, S$ ). To test whether this reflected differential Gli activity on both sides of the ZLI, we observed GFP expression in Tg[GBS::GFP] embryos. At this stage, GFP-positive blood cells were present within the neural tube in all genotypes examined (Fig. 7G-I, green arrowheads point to examples of these GFPpositive blood cells). We found that Gli activity was downregulated in the diencephalon and hypothalamus of $\mathrm{Ftm}^{-1-}$ embryos compared with controls, in the ventral regions (Fig. $7 G, H$ ) as well as on both sides of the ZLI (Fig. 7J-K"). However, Gli activity was not totally absent on both sides of the ZLI (Fig. $7 K-K^{\prime \prime}$ ), as confirmed by quantification of fluorescence intensity (Fig. 7Q). Moreover, in Ftm ${ }^{-1-}$ embryos, the Shh-positive ZLI was wider along the AP axis [Fig. $7 J-K^{\prime}, M, N, P$; width of the ZLI measured at the ventricular surface: $53 \pm 11 \mu \mathrm{m}$ for controls $(n=6)$ and $109 \pm 26 \mu \mathrm{m}$ for $\mathrm{Ftm}^{-1-}(n=4) ; p=$ 0.0095].

In conclusion, in the $\mathrm{Ftm}^{-1-}$ embryos, Shh expression is strongly reduced in the ventral forebrain but maintained and even expanded in the ZLI. Gli activity is dampened in regions adjacent to Shhexpressing domains. The loss of Ftm also uncovered a differential prepattern of Ptch1 and Gli1 expression in different diencephalic prosomeres.

\section{Reintroduction of Gli3R into the Ftm background rescues aspects of the forebrain phenotype}

The impaired production of Gli3R in $\mathrm{Ftm}^{-1-}$ embryos (Vierkotten et al., 2007; Besse et al., 2011) could participate in the observed phenotype. We thus tested how Gli activity in the diencephalon was modified in compound $\left[\mathrm{Ftm}^{-1-} ; \mathrm{Gli3}^{\Delta /+}\right] \mathrm{em}$ bryos, by performing quantification of GFP and Shh expression in $\left[\mathrm{Ftm}^{-/-}\right.$; $\mathrm{Gli}^{\Delta /+}$ ] mutant embryos harboring Tg[GBS::GFP]. The Gli3 ${ }^{\Delta 699}$ allele produces constitutively a short form of Gli3 with partial repressor activity (Hill et al., 2007; Cao et al., 2013). We found that the Shhdependent Gli activity adjacent to the ZLI was reduced in these compound mutants (Fig. 7I, $\left.L-L^{\prime \prime}, Q\right)$. Ptch1 expression in the prethalamus was also downregulated (Fig. $\left.7 O, O^{\prime}, R, S\right)$. Moreover, the increased width of Shh expression in the ZLI was rescued in double mutants [Fig. $7 J-P$; width of the ZLI measured at the ventricular surface: $109 \pm 26$ $\mu \mathrm{m}$ for $\mathrm{Ftm}^{-1-}(n=4)$ and $50 \pm 12 \mu \mathrm{m}$ for $\left(\mathrm{Ftm}^{-/-} \mathrm{Gli}^{\mathrm{\Delta /+}}\right) ; \mathrm{p}=$ 0.028].

We then tested the consequences of Gli3R reintroduction on forebrain patterning and eye formation. ISH for Shh, $\mathrm{Ngn} 2, \mathrm{~Gb} \times 2$,

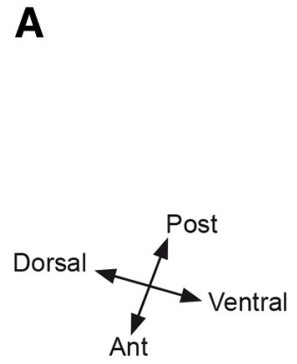

Control
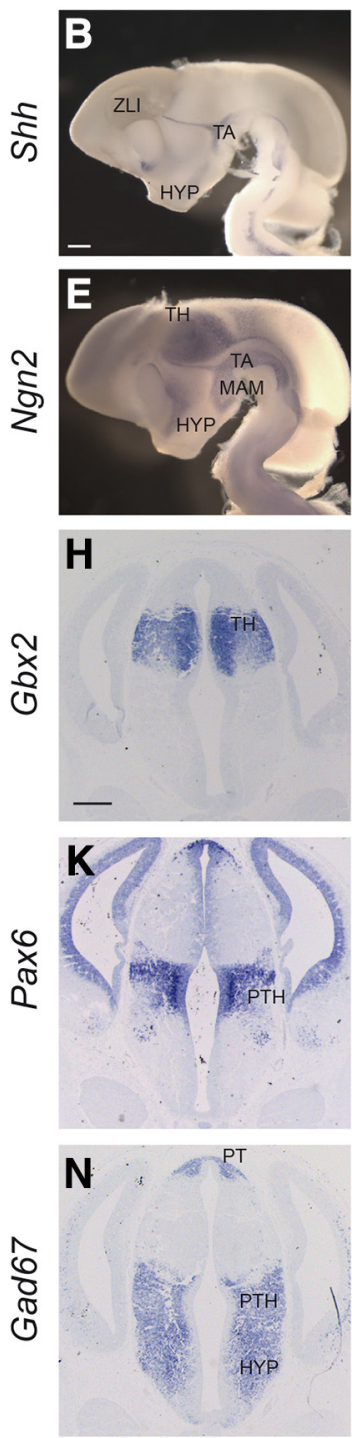

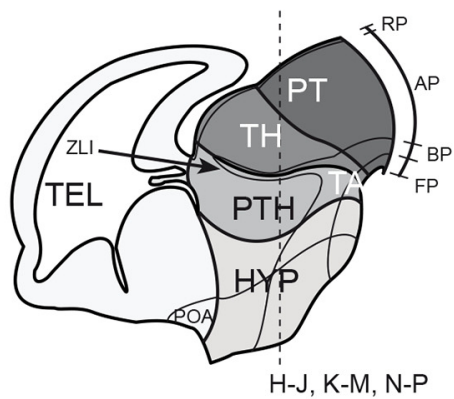

$\mathrm{Ftm}^{-1}$
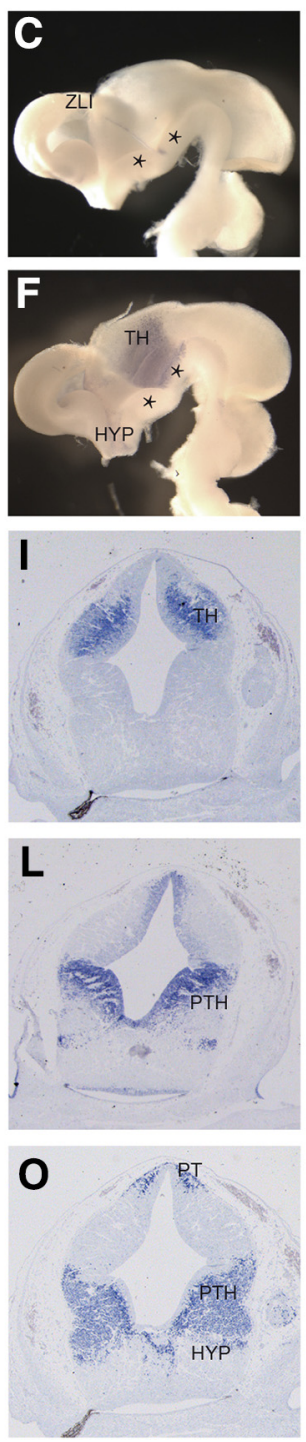

$\mathrm{Ftm}^{-/}, \mathrm{Gli}^{\mathrm{N+}}$
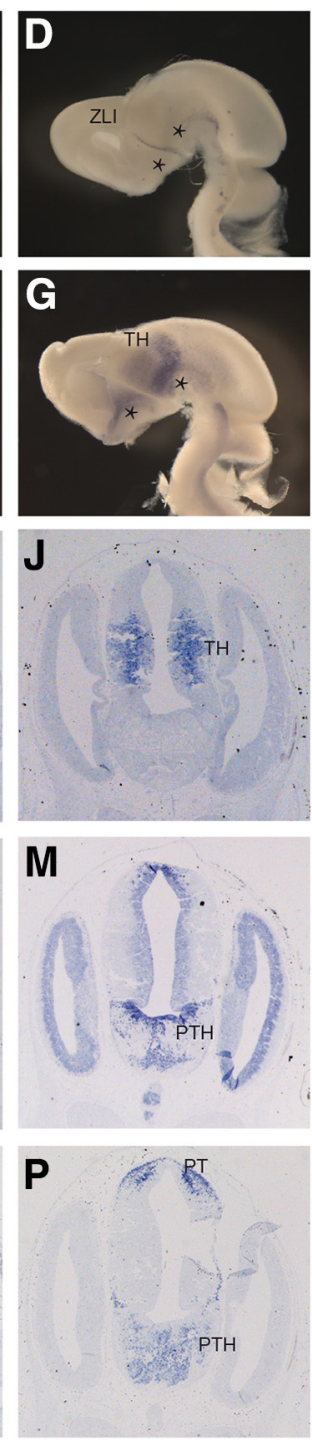

Figure 8. Diencephalon and hypothalamus patterning in compound $\left[\mathrm{Ftm}, \mathrm{Gli3}{ }^{\Delta 699}\right]$ mutants. $\boldsymbol{A}$, Schematic drawings of the E13.5 forebrain in sagittal view. The position of the coronal sections $(\boldsymbol{H}-\boldsymbol{P})$ shown below is indicated with dashed lines. $\boldsymbol{B}-\boldsymbol{G}$, Whole-mount ISH with probes for $\operatorname{Shh}(\boldsymbol{B}-\boldsymbol{D})$ or $\operatorname{Ngn} 2(\boldsymbol{E}-\boldsymbol{G})$ on sagittally-bisected brains viewed from the ventricular side. $\boldsymbol{C}, \boldsymbol{D}, \boldsymbol{F}$, $\boldsymbol{G}$, Black asterisks point to the absence of ventral forebrain. $\boldsymbol{H}-\boldsymbol{P}$, ISH on coronal sections with probe for Gbx2 $(\boldsymbol{H}-\boldsymbol{J})$, Pax6 $(\boldsymbol{K}-\boldsymbol{M})$, or $\mathrm{Gad} 67(\boldsymbol{N}-\boldsymbol{P})$. The genotype of the embryo is indicated at the top. Ant, Anterior; AP, alar plate; BP, basal plate; $\mathrm{FP}$, floor plate; RP, roof plate; Post, posterior; TEL: telencephalon. Scale bars: (in $\boldsymbol{B}$ for coronal sections, in $\boldsymbol{H}$ for whole-mount ISH), $0.5 \mathrm{~mm}$. 
(Fig. $8 C, D$ ). In contrast, optic cup formation was restored in compound mutants, and the optic cup showed correct DV patterning (Fig. $5 C, D, H, I, M, N, R, S$ ). However, the eyes were internalized and brought together in $\left[\mathrm{Ftm}^{-/-} ; \mathrm{Gli}^{\Delta /+}\right]$ embryos, and even more in $\left[\mathrm{Ftm}^{-1-} ; \mathrm{Gli3}^{\Delta / \Delta}\right]$ embryos, and this was associated with a very reduced optic stalk (Fig. $5 C, D, H, I, M, N, R, S$ ). We also analyzed $\left[\mathrm{Ftm}^{+/+} ; \mathrm{Gli3}^{\Delta / \Delta}\right]$ embryos, which looked similar to controls (Fig. $5 E, J, O, T$ ) as found in another study (Christoph Gerhard, University of Düsseldorf, personal communication), indicating that only GliR is required for optic cup formation.

In conclusion, reintroducing Gli3R into the Ftm background rescues some of the defects of $\mathrm{Ftm}^{-1-}$ embryos, such as optic cup agenesis and ZLI enlargement, but not others such as the reduction of the forebrain basal plate and of the rostral thalamus. Moreover, it triggers optic stalk hypoplasia.

\section{The Wnt/ $\beta$-catenin pathway appears unperturbed in the $\mathrm{Ftm}^{-1-}$ embryonic diencephalon}

Perturbations in the $\mathrm{Wnt} / \beta$-catenin pathway have been observed in ciliary gene mutants but their extent and nature depend on the tissue and gene analyzed (Simons et al., 2005; Ocbina et al., 2009; Lancaster et al., 2011). Wnt/ $\beta$-catenin signaling in the diencephalon is involved in specifying thalamic identity and later in promoting formation of the $\mathrm{TH}-\mathrm{C}$ at the expense of TH-R, in parallel to (and independently of) Shh signaling (Braun et al., 2003; Zhou et al., 2004; Bluske et al., 2012). We thus tested whether the activity of the Wnt/ $\beta$-catenin pathway was perturbed in the diencephalon of $\mathrm{Ftm}^{-1-}$ embryos, using Axin2 as a target of the pathway (Bluske et al., 2009). We also examined the expression of $W n t 3 a$ and $W n t 7 b$, two Wnt genes expressed in the developing diencephalon (Bluske et al., 2009). The expression of Axin2 (Fig. 9A, B,G,H,K,L), Wnt3a (Fig. 9C,D,I,J), and Wnt7b (Fig. 9E,F) was similar in control and $\mathrm{Ftm}^{-1-}$ embryos from E10.5 to E13.5, strongly suggesting that the $\mathrm{Wnt} / \beta$-catenin pathway is not perturbed in the $\mathrm{Ftm}^{-/-}$diencephalon.

\section{Cilia of forebrain neural progenitors are severely reduced in number and malformed in $\mathrm{Ftm}^{-/-}$embryos}

In the telencephalon of $\mathrm{Ftm}^{-1-}$ embryos, neural progenitors are devoid of primary cilia (Besse et al., 2011). Because our data indicate that Shh signaling activity is not totally lost in the diencephalon and hypothalamus of $\mathrm{Ftm}^{-1-}$ embryos, we tested the status of cilia in this region. We first analyzed Rpgripll expression in E12.5 controls and found that it was present at the ciliary transition zone in different diencephalic domains including the ZLI (Fig. 10A-C'" ). We then compared cilia in the control and Ftm mutant brain at different stages by immunofluorescence for Arl13b. Arl13b-positive cilia were present in the forebrain of E8.5 control embryos (Fig. 10D- $F^{\prime}$ ) but were not detected in $\mathrm{Ftm}^{-1-}$ embryos (Fig. 10G-I'). In the E12.5 diencephalon, Arl13bpositive cilia were present in the TH, PTH and ZLI in control embryos (Fig. 10J-L) and severely reduced in number in $\mathrm{Ftm}^{-1-}$ embryos (Fig. 10M-O). Arl13b staining was less intense in the remaining cilia (Fig. 10M-O), suggesting that the number of cilia in Ftm mutants might be underestimated using Arl13b as a marker. Indeed, Rpgrip1l is required for Arl13b ciliary localization in several cell types (Wiegering et al., 2018). To further investigate cilium number and shape, we performed scanning electron microscopy (SEM) of the ventricular surface of E13.5 control and $\mathrm{Ftm}^{-1-}$ brains, at different AP levels: in the TH, ZLI, PTH, and HYP (Fig. 10Q- $X$ ). In control embryos, cilia of $\sim 1 \mu \mathrm{m}$ in length were found in the TH, PTH, and ZLI (Fig. $10 P, Q, S, U$, arrows), whereas in the HYP cilia were in average $2 \mu \mathrm{m}$ long (Fig.
$10 P, W$, arrows). Cilia were more difficult to recognize in the ZLI because the ventricular surface of the cells was rich in protrusions and vesicles (Fig. 10S). In the diencephalon and hypothalamus of the $\mathrm{Ftm}^{-1-}$ forebrain, cilia were in majority absent or reduced to button-like structures (Fig. $10 R, T, V, X$, arrowheads), with a few very long cilia often abnormal in shape (Fig. $10 R, T, V, X$, arrows). These remaining cilia were present in all regions, but more frequently in the ZLI (Fig. 10T).

In conclusion, cilia were absent from the forebrain of $\mathrm{Ftm}^{-1-}$ embryos as soon as E8.5. At E12.5 they were reduced in number in the diencephalon and hypothalamus, and the remaining cilia were longer than in controls and often presented an abnormal shape.

\section{Discussion}

The role of cilia in the forebrain has been little studied outside of the telencephalon. In this paper we have studied the role of the Ftm/Rpgrip1l ciliopathy gene in patterning of the diencephalon, hypothalamus, and eyes. At the end of gestation, $\mathrm{Ftm}^{-1-}$ fetuses displayed anophthalmia, reduction of the ventral hypothalamus and disorganization of diencephalic nuclei and axonal tracts. We examined the developmental defects underlying this phenotype. $\mathrm{Ftm}^{-1-}$ embryos showed a severe reduction of ventral forebrain structures accompanied by a dorsoventral expansion of alar diencephalic domains and a loss of the rostral thalamus (Fig. 11A). Optic vesicles formed but optic cup morphogenesis did not occur. Investigating the molecular mechanisms of these defects, we uncovered region-specific perturbations of the $\mathrm{Hh} / \mathrm{Gli}$ pathway, whereas the Wnt/ $\beta$-catenin pathway appeared unaltered. Combined with our previous studies (Besse et al., 2011; Laclef et al., 2015), our data lead to a global understanding of the role of primary cilia in forebrain patterning and morphogenesis of their relationship with Shh signaling.

Do the forebrain defects of Ftm mutants correspond to a ciliary phenotype? Apart from the disorganization of the diencephalictelencephalic boundary and TCA tracts (Willaredt et al., 2008, 2013; Magnani et al., 2015), the defects observed in this study have not been reported in other ciliary mutants, some of which die too early (Gorivodsky et al., 2009). It was thus important to study the number and integrity of cilia in the diencephalon and hypothalamus of $\mathrm{Ftm}^{-1-}$ embryos at different stages. We found a near-total loss of cilia in the progenitors of the forebrain of $\mathrm{Ftm}^{-1-}$ embryos at E8.5. At E12.5, cilia were severely reduced in number, and their shape and content were highly abnormal. This, combined with our previous studies (Besse et al., 2011), strongly suggests that the forebrain defects observed in Ftm mutants are because of the ciliary defects in neural progenitors.

Our data point to region-specific defects in Shh signaling in the forebrain of Ftm mutants. The reduction in ventral forebrain areas and the loss of the TH-R in Ftm mutants suggest an impaired response to Shh signals, similar to what has been previously observed in the ventral spinal cord of these mutants (Vierkotten et al., 2007). Indeed, tegmental areas of the diencephalon and hypothalamus depend on Shh signaling from the notochord and prechordal plate as soon as E7.5, which induces Shh expression in the forebrain midline. Neural Shh is in turn required from E8.5 onward for correct formation of the basal diencephalon and hypothalamus (Dale et al., 1997; Szabó et al., 2009a,b; Shimogori et al., 2010; Zhao et al., 2012). Later, at E10.5-E12.5, high Hh/Gli activity (from the ZLI and the ventral forebrain) is required for the formation of the TH-R, whereas the $\mathrm{TH}-\mathrm{C}$ requires lower $\mathrm{Hh} / \mathrm{Gli}$ activity (Hashimoto-Torii et al., 2003; Jeong et al., 2011). Thus, our observation of the loss of the 


\section{E11.5}
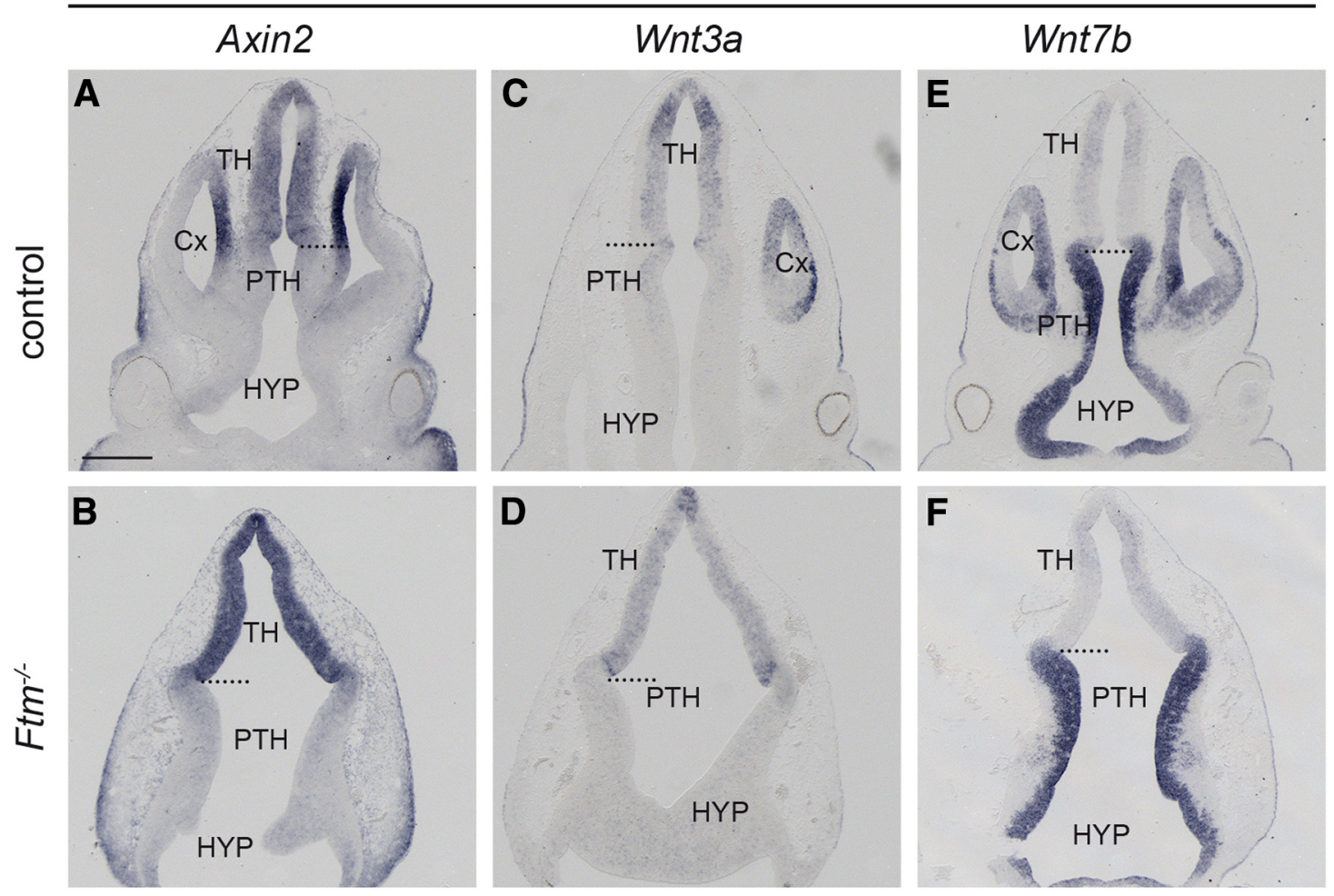

E10.5
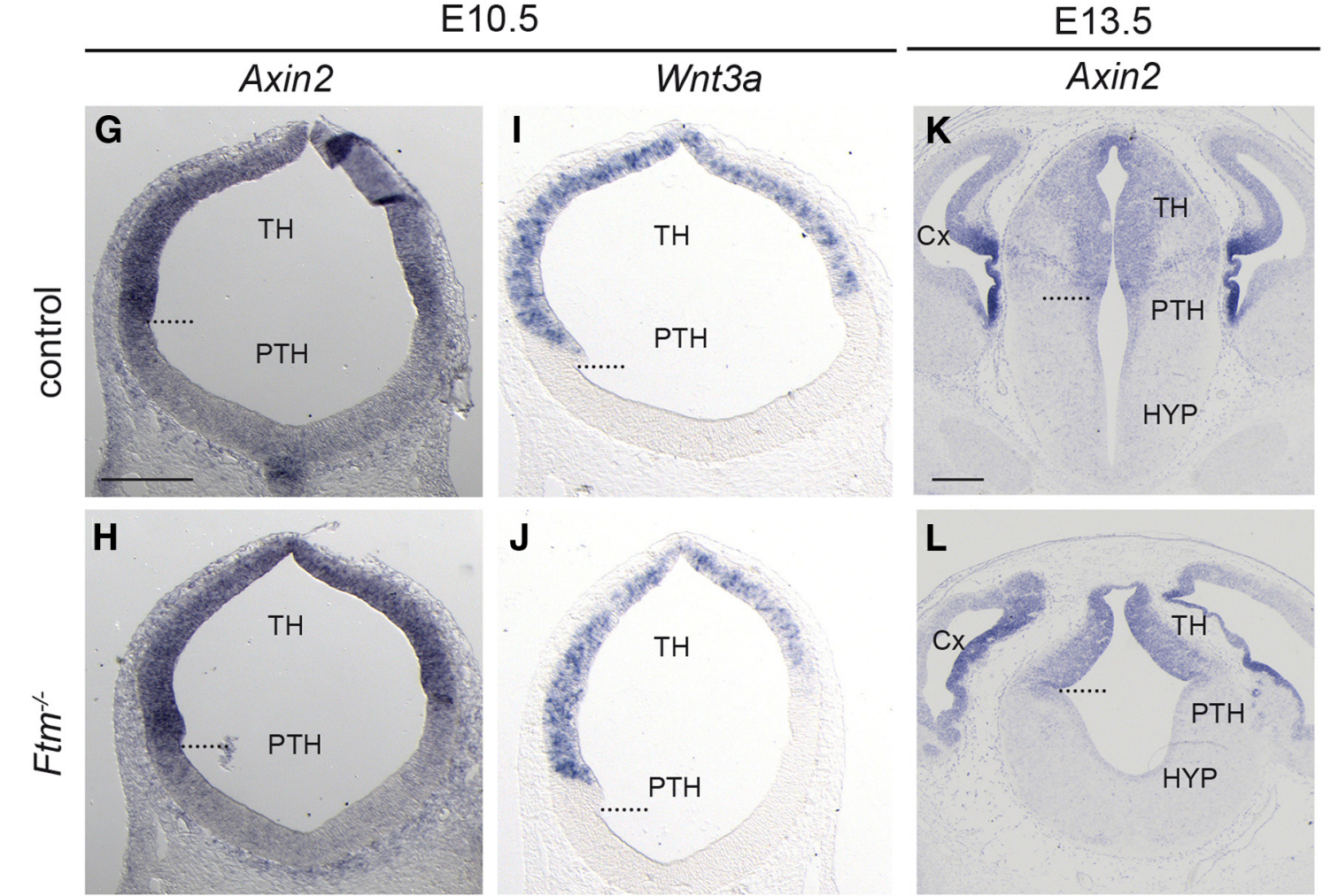

Figure 9. Wnt/ $\beta$-catenin pathway activity in the forebrain of $F t m$ mutants. ISH with probes for $A x i n 2(\boldsymbol{A}, \boldsymbol{B}, \mathbf{G}, \boldsymbol{H}, \boldsymbol{K}, \boldsymbol{L}), \boldsymbol{W n t} 3 a(\boldsymbol{C}, \boldsymbol{D}, \boldsymbol{I}, \boldsymbol{J})$, and $W n t 7 b(\boldsymbol{E}, \boldsymbol{F})$ on coronal sections of E11.5 $(\boldsymbol{A}-\boldsymbol{F}), \mathrm{E} 10.5$ $(\mathbf{G}-\boldsymbol{J})$, and $\mathbf{E 1 3 . 5}(\boldsymbol{K}, \boldsymbol{L})$ embryos at the level of the forebrain. The genotype is indicated on the left of the Figure. The dotted lines indicate the position of the ZLI. Scale bars: (in $\boldsymbol{A}) \boldsymbol{A}-\boldsymbol{F}$, (in $\boldsymbol{G}$ ) $\mathbf{G}-\boldsymbol{J}$, (in K) $K, L, 0.5 \mathrm{~mm}$.

TH-R and the expansion of the TH-C in $\mathrm{Ftm}^{-1-}$ embryos is totally consistent with the strong reduction of GliA activity as assayed by Tg[GBS::GFP] and the near-total absence of Shh expression in the ventral diencephalon at E12.5.
However, the phenotype of the Ftm mutant in the forebrain differs from that of a Shh mutant. In $S h h^{-1-}$ embryos, unlike in $\mathrm{Ftm}^{-1-}$ embryos, the whole diencephalon is extremely reduced in size because of reduced proliferation and survival as soon as the 
Shh/Arl13b/Rpgrip1l/DAPI - E12.5
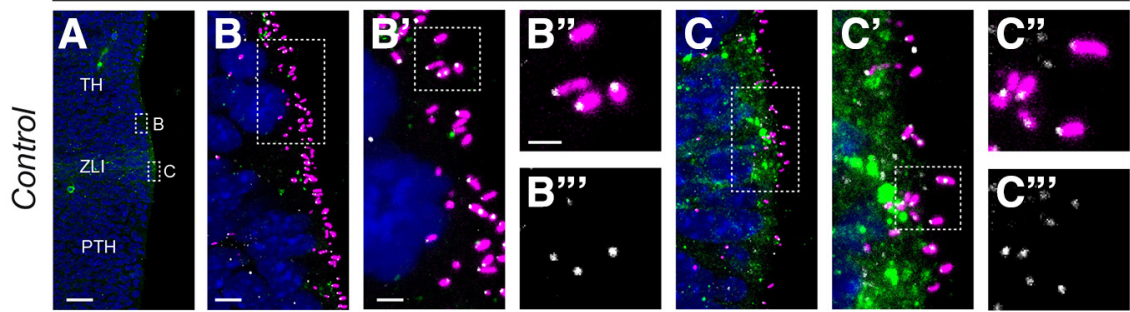

Shh/Arl13b-E8.5
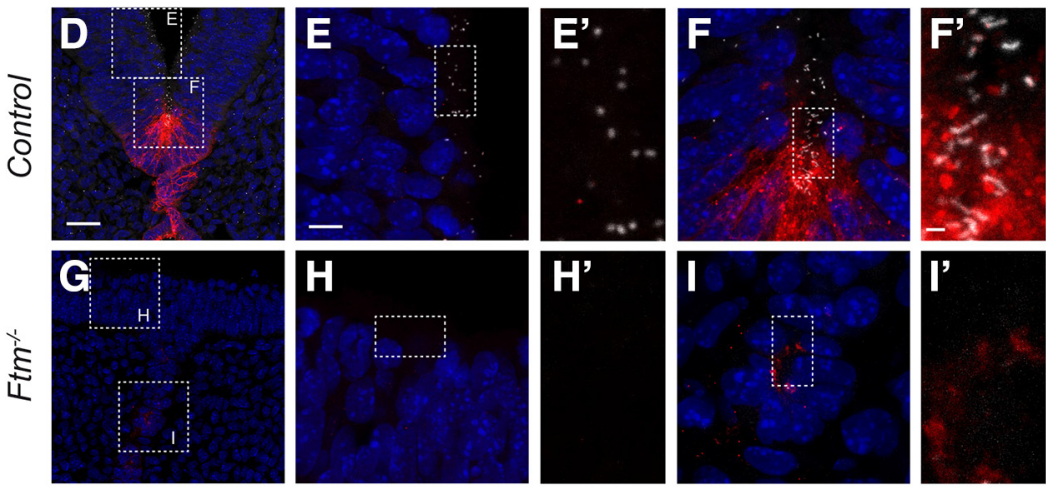

$\mathbf{P}$
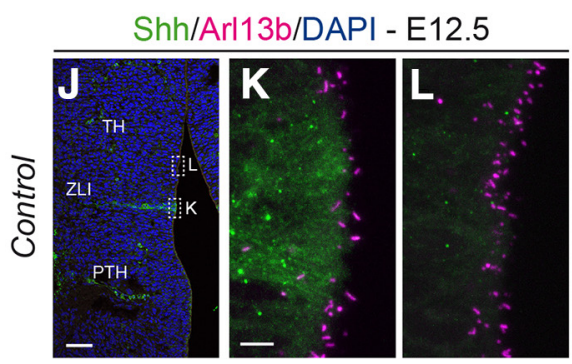

$\left.{ }^{0.15}\right]$

CILIA DENSITY
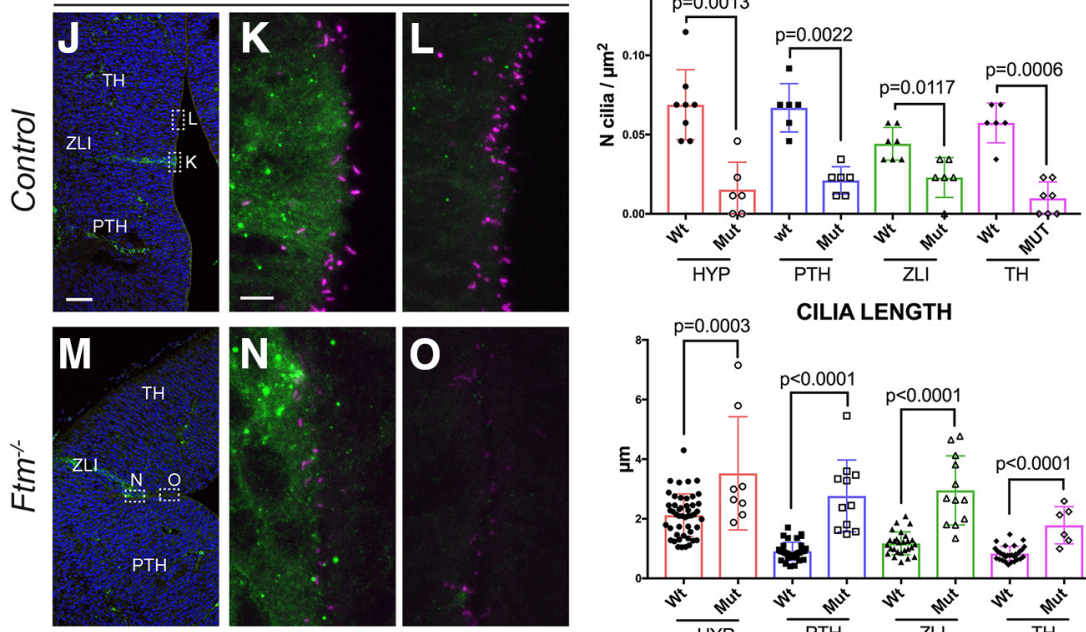

CILIA LENGTH

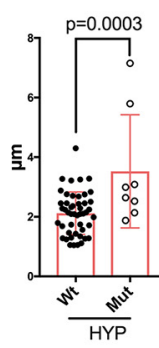

SEM-E13.5
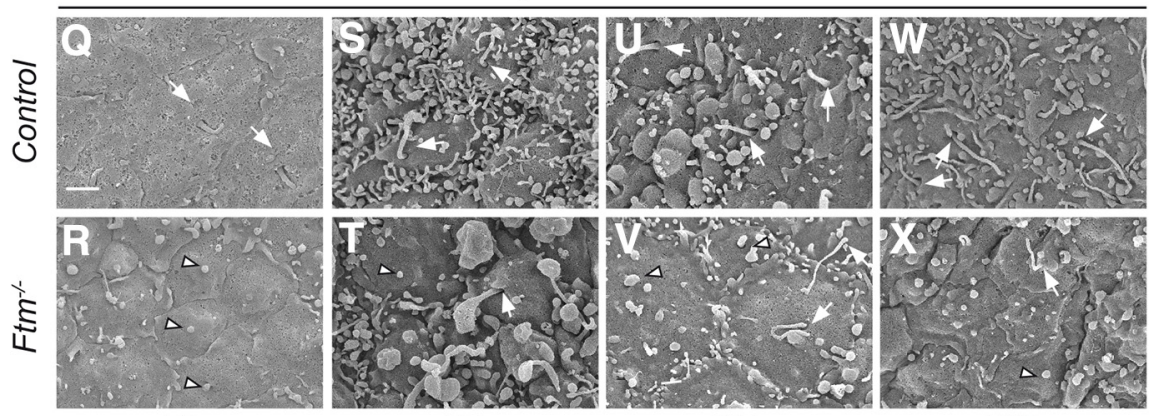

ZLI

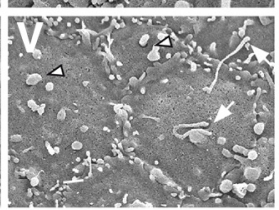

PTH

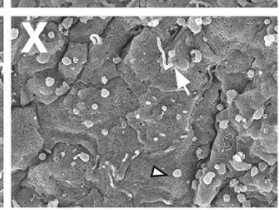

HYP

Figure 10. Cilia in the forebrain of Ftm mutants. $\boldsymbol{A}-\boldsymbol{C}^{\prime \prime}$, Immunofluorescence on coronal sections of E12.5 control embryos with antibodies for Shh (green), Arl13b (magenta), and Rpgrip1l (white). Nuclei are stained with DAPI. $\boldsymbol{B}^{\prime \prime \prime}, \boldsymbol{C}^{\prime \prime}$, , Only Rpgrip1l is shown. $\boldsymbol{D}-\boldsymbol{I}^{\prime}$, Immunofluorescence on coronal sections of E8.5 control $\left(\boldsymbol{D}-\boldsymbol{F}^{\prime}\right)$ and $F \mathrm{tm}^{-I-}\left(\boldsymbol{G}-\boldsymbol{I}^{\prime}\right)$ embryos with antibodies for Shh (red) and Arl13b (white). Nuclei are stained with DAPI. $\boldsymbol{D}, \boldsymbol{G}$, White squares indicate the regions magnified in $\boldsymbol{E}, \boldsymbol{F}$, and $\boldsymbol{H}, \boldsymbol{I}$, respectively. $\boldsymbol{E}-\boldsymbol{I}$, White rectangles indicate the regions magnified in $\boldsymbol{E}^{\prime}-\boldsymbol{I}^{\prime}$, respectively. $\boldsymbol{J}-\mathbf{0}$, Immunofluorescence on coronal sections of E12.5 control $(\boldsymbol{J}-\boldsymbol{L})$ and $F t m^{-/-}(\boldsymbol{M}-\mathbf{0})$ embryos with antibodies for Shh (green) and Arl13b (magenta). $\boldsymbol{J}, \boldsymbol{M}$, Nuclei are stained with DAPI. White rectangles indicate the regions magnified in $\boldsymbol{K}, \boldsymbol{L}$ and $\boldsymbol{N}, \boldsymbol{0}$, respectively. $\boldsymbol{P}$, Graph comparing the density (top) and length (bottom) of cilia on the SEM images, in the HYP, PTH, TH, and ZLI regions of control and Ftm ${ }^{-1-}$ embryos. $\mathbf{Q}-\boldsymbol{X}$, SEM of the

15 s stage (Ishibashi and McMahon, 2002). Moreover, contrary to Shh mutants (Chiang et al., 1996), Ftm mutants never show cyclopia, even when two copies of Gli3R are reintroduced. This suggests that a low level of Hh/Gli pathway activity (undetected by the GBS::GFP transgene) sufficient to separate the eye fields and to promote forebrain morphogenesis is produced from the underlying prechordal mesendoderm of $\mathrm{Ftm}^{-1-}$ embryos. Our observation of sparse Ptch1- and Shhpositive cells in the neural plate of E8.0 E8.5 mutant embryos consolidates this assumption.

In that respect, examination of $\mathrm{Hh} / \mathrm{Gli}$ activity at the ZLI is very informative. Indeed, the ZLI forms in $\mathrm{Ftm}^{-1-}$ embryos and is even wider than in controls. This widening is accounted for by the reduction in Gli3R levels, because it is rescued in compound $\left[\mathrm{Ftm}^{-/-}, \mathrm{Gli3}^{\Delta /+}\right] \mathrm{em}$ bryos. Consistent with these data, Gli3 repression by Wnt signals is required for controlling the width of the ZLI in chicken embryos (Martinez-Ferre et al., 2013). Moreover, Shh from the ZLI appears to be able to signal, although with lower efficiency than in controls. Thus, the Hh/Gli pathway is still active in $\mathrm{Ftm}^{-1-}$ embryos.

The ZLI has been proposed, initially in chick, to form through an inductive process requiring Shh signaling from the diencephalic basal plate (Kiecker and Lumsden, 2004; Zeltser, 2005; Epstein, 2012). In mouse mutants in which expression of a functional Shh is absent from the ventral diencephalon, the ZLI does not form (Szabó et al., 2009b). If ZLI formation requires Shh signals from the basal plate, how can it occur in Ftm mutants, which display no Shh expression in the basal diencephalon? In E9.5 $\mathrm{Ftm}^{-1-}$ embryos, a discrete patch of Shh expression remained in the basal plate at the level of the future ZLI. We propose that this patch of Shh expression is sufficient for the initiation of ZLI formation in Ftm mutants. Because this patch does not give rise to detectable Gli activity, we speculate that here Shh might signal through Gliindependent, non-canonical pathway (Carballo et al., 2018). Alternatively, re-

ventricular surface in different regions of control $(\boldsymbol{Q}, \boldsymbol{S}, \boldsymbol{U}, \boldsymbol{W})$ and $\mathrm{Ftm}^{-1-}(\boldsymbol{R}, \boldsymbol{T}, \boldsymbol{V}, \boldsymbol{X})$ hemisected brains. White arrows point to the base of cilia, white arrowheads point to buttonlike structures surrounded by a ciliary pocket, similar to those found in the cortex of $\mathrm{Ftm}^{-1-}$ embryos (Besse et al., 2011). Scale bars: (in $\boldsymbol{A}, \boldsymbol{J}) \boldsymbol{A}, \boldsymbol{J}, \boldsymbol{M}, 50 \mu \mathrm{m}$; (in $\boldsymbol{D}) \boldsymbol{D}, \boldsymbol{G}, 20 \mu \mathrm{m}$; (in $\boldsymbol{B}) \boldsymbol{B}$, C, (in $\boldsymbol{E}) \boldsymbol{E}, \boldsymbol{F}, \boldsymbol{H}, \boldsymbol{I}, 5 \mu \mathrm{m}$; (in $\left.\boldsymbol{B}^{\prime}\right) \boldsymbol{B}^{\prime}, \boldsymbol{C}^{\prime}$, (in $\left.\boldsymbol{K}\right) \boldsymbol{K}, \boldsymbol{L}, \boldsymbol{N}, \mathbf{O}, 2 \mu \mathrm{m}$; (in $\left.\boldsymbol{B}^{\prime \prime}\right) \boldsymbol{B}^{\prime \prime}, \boldsymbol{B}^{\prime \prime \prime}, \boldsymbol{C}^{\prime \prime}, \boldsymbol{C}^{\prime \prime \prime}$, (in $\left.\boldsymbol{F}^{\prime}\right) \boldsymbol{F}^{\prime}, \boldsymbol{G}^{\prime}, \boldsymbol{H}, \boldsymbol{I}^{\prime}$, (in $\left.\mathbf{Q}\right) \mathbf{Q}-\boldsymbol{X}, 1 \mu \mathrm{m}$. 

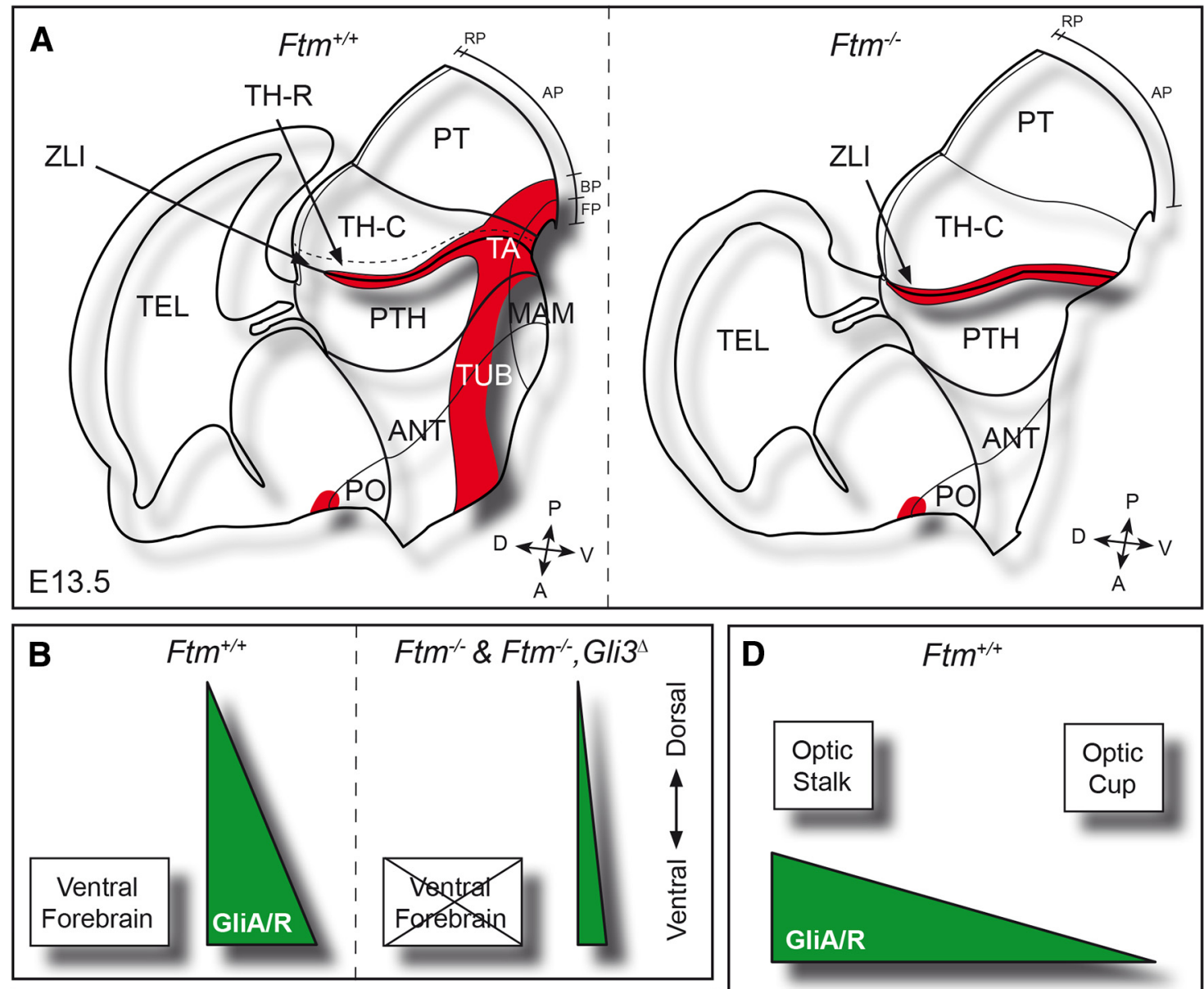

D $\quad \mathrm{Ftm}^{+/+}$
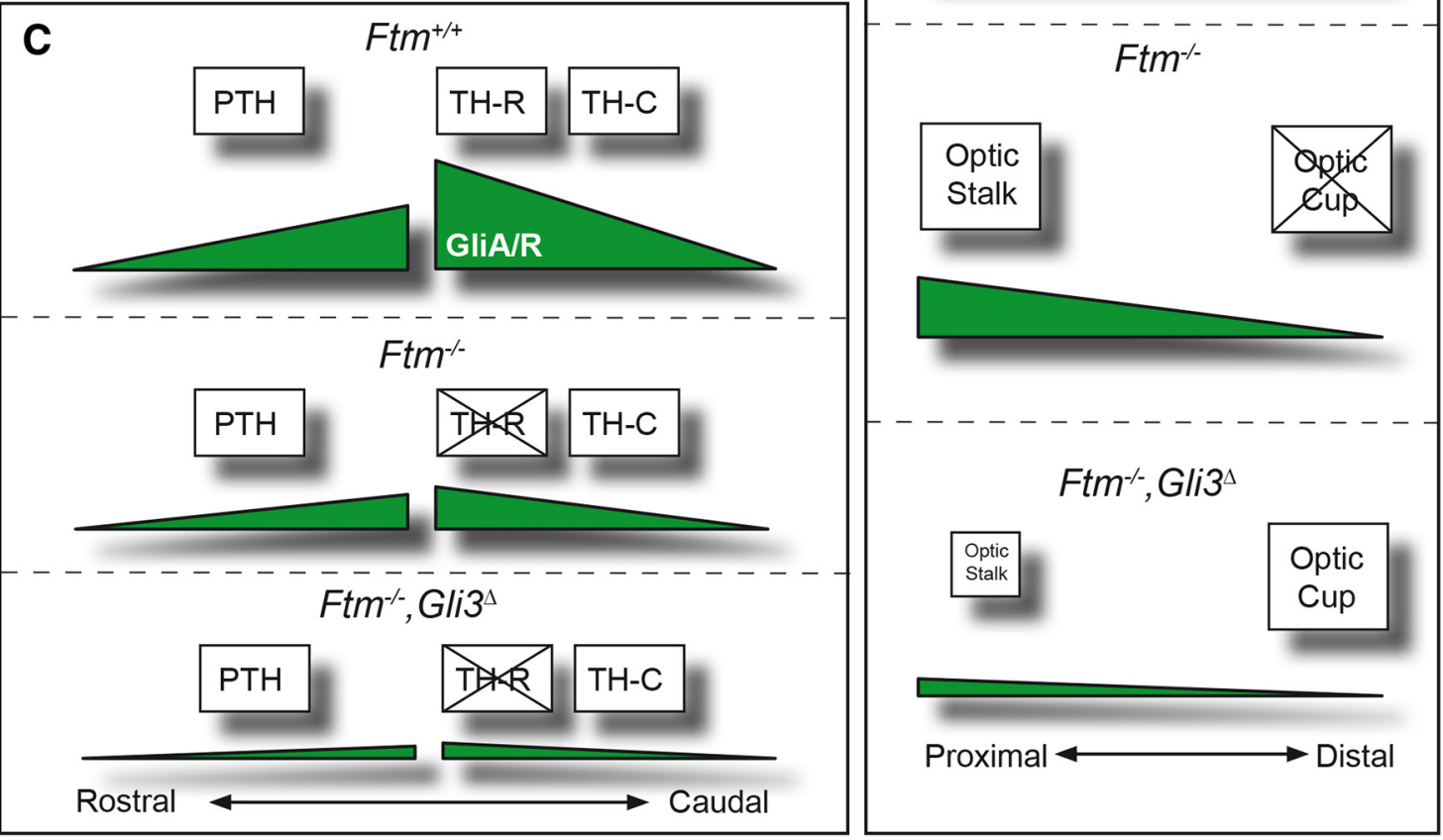

Figure 11. Schematics of forebrain patterning defects in Ftm embryos and their link to perturbations of Gli activity. $\boldsymbol{A}$, Schematic drawings of the forebrain of $\mathrm{E} 13.5 \mathrm{control}$ (left) and $\mathrm{Ftm}{ }^{-1-}$ (right) embryos. Shh expression domains are in red. $\boldsymbol{B}-\boldsymbol{D}$, Interpretive schematics of the $\mathrm{Gli} / \mathrm{G} / \mathrm{G}$ r ratios (green) during ventral forebrain formation $(\boldsymbol{B})$, alar diencephalon patterning $(\boldsymbol{C})$, and optic vesicle patterning into optic stalk and optic cup $(\boldsymbol{D})$ in control, $\mathrm{Ftm}^{-/-}$and $\left[\mathrm{Ftm}^{-1-}, \mathrm{Gli}^{\Delta}\right]$ embryos. $\boldsymbol{B}$, From E8.0 onward, a high GliA/GliR ratio is required for the formation of the ventral forebrain. In Ftm ${ }^{-1-}$ as well as in compound $\left[\mathrm{Ftm}^{-1-}, \mathrm{Gli}^{\Delta}\right.$ ] embryos, the reduction this ratio causes a strong reduction of the ventral forebrain. $\boldsymbol{C}$, At later stages (E10.5-12.5), in the alar diencephalon, a high GliA/GliR ratio is required for TH-R formation, whereas a lower ratio is sufficient for PTH and TH-C formation. In Ftm ${ }^{-1-}$ embryos the TH-R is lost but the ratio is sufficient for PTH and TH-C formation. D, From E9.0 onward, in the optic vesicle, optic stalk formation requires a high GliA/GliR ratio, while the optic cup requires that only GliR is present. Low levels of GliA are sufficient for optic stalk formation in Ftm ${ }^{-/-}$embryos. In contrast, the optic cup is not formed because of the reduction of GliR levels. In compound [Ftm ${ }^{-1-}, \mathrm{Gli3}^{\Delta}$ ] embryos, the optic cup is rescued and the optic stalk is reduced (the eyes are closer to one another) because of the reintroduction of Gli3R. A, Anterior; $\mathrm{AP}$, alar plate; $\mathrm{BP}, \mathrm{basal}$ plate; $\mathrm{D}$, dorsal; $\mathrm{FP}$, floor plate; $\mathrm{P}$, posterior; $\mathrm{PO}$, preoptic area; RP, roof plate; TEL, telencephalon; $\mathrm{V}$, ventral. 
maining cilia in the prospective ZLI could be still functional and could lead to a low level of Gli activity (undetected in our study) sufficient to initiate ZLI formation.

Examination of the eye in Ftm mutants provides another example of the region-specific functions of cilia. In $\mathrm{Ftm}^{-1-} \mathrm{em}-$ bryos, the optic cup and lens are totally absent. Gli3 is known to be involved in optic cup formation (Furimsky and Wallace, 2006), but it was not known so far whether it acted as a repressor or as an activator. We found optic cups with correct DV patterning in compound $\left[\mathrm{Ftm}^{-1-}, \mathrm{Gli3}^{\Delta /+}\right]$ and $\left[\mathrm{Ftm}^{-1-}, \mathrm{Gli}^{\Delta / \Delta}\right] \mathrm{em}-$ bryos, showing that Gli3R, and not Gli3A, is crucial for optic cup formation, and that the function of cilia in this process is mediated by Gli3R. This was confirmed by the analysis of $\left[\mathrm{Ftm}^{+/+}\right.$, $\mathrm{Gli3}^{\Delta / \Delta}$ ] siblings, which displayed normal retina. The retinal phenotype of Ftm mutants is reminiscent to that of the telencephalon, where dorsal structures are reduced because of the reduction in Gli3R levels (Besse et al., 2011; Laclef et al., 2015). However, in compound $\left[\mathrm{Ftm}^{-1-}, \mathrm{Gli}^{\Delta /+}\right]$ and $\left[\mathrm{Ftm}^{-1-}, \mathrm{Gli}^{\Delta / \Delta}\right]$ embryos, the optic cups were closer to each other under the ventral forebrain and even partially fused in some cases, and the optic stalk was almost totally absent. Thus, cilia are required both for GliRdependent optic cup formation and for GliA-dependent optic stalk morphogenesis.

In conclusion, our data show that, in Ftm mutants, forebrain structures requiring high GliA activity, such as the rostral thalamus and ventral forebrain, and structures that require high GliR activity, such as the optic cup, are lost. In contrast, structures that require low or intermediate $\mathrm{Hh} / \mathrm{Gli}$ activity, such as $\mathrm{TH}-\mathrm{C}$ or the optic stalk, are still present. Thus, different regions of the forebrain are differently affected by the loss of cilia depending on their specific requirement for GliA or GliR activity (Fig. 11B-D).

Are our data relevant for human disease? There are few reports of hypothalamic or diencephalic malformations in ciliopathies. However, a precise analysis of the forebrain is rarely possible in fetuses with severe ciliopathies such as Meckel syndrome. Microphthalmia and benign tumors called diencephalic or hypothalamic hamartomas have been observed in Meckel syndrome and other ciliopathies (Ahdab-Barmada and Claassen, 1990; Roume et al., 1998; Paetau et al., 2008; Poretti et al., 2011, 2017; Del Giudice et al., 2014). Interestingly, diencephalic hamartomas have been linked to mutations in GLI3 and other SHH pathway genes (Shin et al., 1999; Hildebrand et al., 2016), suggesting that those observed in ciliopathies could also be caused by defects in SHH signaling. Holoprosencephaly is rarely described in ciliopathies, and only in the most severe form, Meckel syndrome (Paetau et al., 1985, Ahdab-Barmada and Claassen, 1990). This may be surprising, given the essential role of cilia in vertebrate Shh signaling. Our study of the forebrain of Ftm mutants provides a potential explanation, as we find clear phenotypic differences between the Ftm mutants and $\mathrm{Hh} / \mathrm{Gli}$ pathway mutants. Nevertheless, ciliopathy genes seem to play a role as modifier genes for HPE. HPE shows high phenotypic variability in single families, which has led to the proposal that a combination of mutations in HPE genes could account for the variable severity of the phenotype (the multi-hit hypothesis). In favor of this hypothesis, digenic or oligogenic inheritance has been identified in several HPE families (Mouden et al., 2016; Kim et al., 2019). Interestingly, homozygous mutations in the STIL gene encoding a pericentriolar and centrosomal protein have been found in patients with HPE and microcephaly (Mouden et al., 2015, Kakar et al., 2015). Mouse Stil ${ }^{-1-}$ embryos display severe forebrain midline defects (Izraeli et al., 1999) and ciliogenesis, centriole duplication and Shh signaling are defective in the absence of STIL
(David et al., 2014; Mouden et al., 2015), further suggesting an involvement of cilia defects in HPE.

More generally, our study of the ciliopathy gene mutant Rpgrip1l/Ftm calls for further examination of ciliary and ciliopathy genes in human neurodevelopmental diseases associated with SHH signaling defects.

\section{References}

Ahdab-Barmada M, Claassen D (1990) A distinctive triad of malformations of the central nervous system in the meckel-gruber syndrome. J Neuropathol Exp Neurol 49:610-620.

Alvarez-Bolado G, Paul FA, Blaess S (2012) Sonic hedgehog lineage in the mouse hypothalamus: from progenitor domains to hypothalamic regions. Neural Dev 7:4.

Anselme I, Laclef C, Lanaud M, Rüther U, Schneider-Maunoury S (2007) Defects in brain patterning and head morphogenesis in the mouse mutant Fused toes. Dev Biol 304:208-220.

Arts HH, Doherty D, van Beersum SE, Parisi MA, Letteboer SJ, Gorden NT, Peters TA, Märker T, Voesenek K, Kartono A, Ozyurek H, Farin FM, Knoers HY, Wolfrum U, Brunner HG, Cremers FP, Glass IA, Knoers NV, Roepman R (2007) Mutations in the gene encoding the basal body protein RPGRIP1L, a nephrocystin-4 interactor, cause joubert syndrome. Nat Genet 39:882-888.

Balaskas N, Ribeiro A, Panovska J, Dessaud E, Sasai N, Page KM, Briscoe J, Ribes V (2012) Gene regulatory logic for reading the sonic hedgehog signaling gradient in the vertebrate neural tube. Cell 148:273-284.

Benadiba C, Magnani D, Niquille M, Morlé L, Valloton D, Nawabi H, AitLounis A, Otsmane B, Reith W, Theil T, Hornung JP, Lebrand C, Durand B (2012) The ciliogenic transcription factor RFX3 regulates early midline distribution of guidepost neurons required for corpus callosum development. PLoS Genet 8:e1002606.

Besse L, Neti M, Anselme I, Gerhardt C, Rüther U, Laclef C, SchneiderMaunoury S (2011) Primary cilia control telencephalic patterning and morphogenesis via Gli3 proteolytic processing. Development 138:20792088 .

Blaess S, Szabó N, Haddad Tóvolli R, Zhou X, Álvarez-Bolado G (2015) Sonic hedgehog signaling in the development of the mouse hypothalamus. Front Neuroanat 8:156.

Bluske KK, Kawakami Y, Koyano-Nakagawa N, Nakagawa Y (2009) Differential activity of Wnt/beta-catenin signaling in the embryonic mouse thalamus. Dev Dyn 238:3297-3309.

Bluske KK, Vue TY, Kawakami Y, Taketo MM, Yoshikawa K, Johnson JE, Nakagawa Y (2012) $\beta$-Catenin signaling specifies progenitor cell identity in parallel with shh signaling in the developing mammalian thalamus. Development 139:2692-2702.

Böse J, Grotewold L, Rüther U (2002) Pallister-Hall syndrome phenotype in mice mutant for Gli3. Hum Mol Genet 11:1129-1135.

Braun MM, Etheridge A, Bernard A, Robertson CP, Roelink H (2003) Wnt signaling is required at distinct stages of development for the induction of the posterior forebrain. Development 130:5579-5587.

Cao T, Wang C, Yang M, Wu C, Wang B (2013) Mouse limbs expressing only the Gli3 repressor resemble those of sonic hedgehog mutants. Dev Biol 379:221-228.

Carballo GB, Honorato JR, de Lopes GPF, Spohr TCLSE (2018) A highlight on sonic hedgehog pathway. Cell Commun Signal 16:11.

Chiang C, Litingtung Y, Lee E, Young KE, Corden JL, Westphal H, Beachy PA (1996) Cyclopia and defective axial patterning in mice lacking Sonic hedgehog gene function. Nature 383:407-413.

Dale JK, Vesque C, Lints TJ, Sampath TK, Furley A, Dodd J, Placzek M (1997) Cooperation and BMP7 and SHH in the induction of forebrain ventral midline cells by prochordal mesoderm. Cell 90:257-269.

David A, Liu F, Tibelius A, Vulprecht J, Wald D, Rothermel U, Ohana R, Seitel A, Metzger J, Ashery-Padan R, Meinzer HP, Gröne HJ, Izraeli S, Krämer A (2014) Lack of centrioles and primary cilia in STIL $(-/-)$ mouse embryos. Cell Cycle 13:2859-2868.

Del Giudice E, Macca M, Imperati F, D’Amico A, Parent P, Pasquier L, Layet V, Lyonnet S, Stamboul-Darmency V, Thauvin-Robinet C, Franco B; Oral-Facial-Digital Type I (OFD1) Collaborative Group (2014) CNS involvement in OFD1 syndrome: a clinical, molecular, and neuroimaging study. Orphanet J Rare Dis 9:74.

Delogu A, Sellers K, Zagoraiou L, Bocianowska-Zbrog A, Mandal S, Guimera J, Rubenstein JL, Sugden D, Jessell T, Lumsden A (2012) Subdortical 
visual shell nuclei targeted by ipRGCs develop from a Sox14+GABAergic progenitor and require Sox14 to regulate daily activity rhythms. Neuron 75:648-662.

Delous M, Baala L, Salomon R, Laclef C, Vierkotten J, Tory K, Golzio C, Lacoste T, Besse L, Ozilou C, Moutkine I, Hellman NE, Anselme I, Silbermann F, Vesque C, Gerhardt C, Rattenberry E, Wolf MT, Gubler MC, Martinovic J, et al. (2007) The ciliary gene RPGRIP1L is mutated in cerebello-oculo-renal syndrome (Joubert syndrome type B) and meckel syndrome. Nat Genet 39:875-881.

Epstein DJ (2012) Regulation of thalamic development by Sonic hedgehog. Front Neurosci 6:57.

Fernandes M, Hébert JM (2008) The ups and downs of holoprosencephaly: dorsal versus ventral patterning forces. Clin Genet 73:413-423.

Fode C, Ma Q, Casarosa S, Ang SL, Anderson DJ, Guillemot F (2000) A role for neural determination genes in specifying the dorsoventral identify of telencephalic neurons. Genes Dev 14:67-80.

Furimsky M, Wallace VA (2006) Complementary gli activity mediates early patterning of the mouse visual system. Dev Dyn 235:594-605.

Garel S, Marín F, Mattéi MG, Vesque C, Vincent A, Charnay P (1997) Family of Ebf/Olf-1-related genes potentially involved in neuronal differentiation and regional specification in the central nervous system. Dev Dyn 210:191-205.

Gerhardt C, Lier JM, Burmühl hl S, Struchtrup A, Deutschmann K, Vetter M, Leu T, Reeg S, Grune T, Rüther U (2015) The transition zone protein Rpgrip1l regulates proteasomal activity at the primary cilium. J Cell Biol 210:115-133.

Goetz SC, Anderson KV (2010) The primary cilium: a signaling centre during vertebrate development. Nat Rev Genet 11:331-344.

Gorivodsky M, Mukhopadhyay M, Wilsch-Braeuninger M, Phillips M, Teufel A, Kim C, Malik N, Huttner W, Westphal H (2009) Intraflagellar transport protein 172 is essential for primary cilia formation and plays a vital role in patterning the mammalian brain. Dev Biol 325:24-32.

Haddad-Tóvolli R, Heide M, Zhou X, Blaess S, Alvarez-Bolado G (2012) Mouse thalamic differentiation: Gli-dependent and Gli-independent prepattern. Front Neurosci 6:27.

Haddad-Tóvolli R, Paul FA, Zhang Y, Zhou X, Theil T, Puelles L, Blaess S, Alvarez-Bolado G (2015) Differential requirements for Gli2 and Gli3 in the regional specification of the mouse hypothalamus. Front Neuroanat 9:34.

Hagemann AI, Scholpp S (2012) The tale of the three brothers: Shh, Wnt, and Fgf during development of the thalamus. Front Neurosci 6:76.

Hallonet M, Kaestner KH, Martin-Parras L, Sasaki H, Betz UA, Ang SL (2002) Maintenance of the specification of the anterior definitive endoderm and forebrain depends on the axial mesendoderm: a study using HNF3 $\beta /$ Foxa 2 conditional mutants. Dev Biol 243:20-33.

Han YG, Spassky N, Romaguera-Ros M, Garcia-Verdugo JM, Aguilar A, Schneider-Maunoury S, Alvarez-Buylla A (2008) Hedgehog signaling and primary cilia are required for the formation of adult neural stem cells. Nat Neurosci 11:277-284.

Hashimoto-Torii K, Motoyama J, Hui CC, Kuroiwa A, Nakafuku M, Shimamura K (2003) Differential activities of sonic hedgehog mediated by gli transcription factors define distinct neuronal subtypes in the dorsal thalamus. Mech Dev 120:1097-1111.

Hevner RF, Miyashita-Lin E, Rubenstein JL (2002) Cortical and thalamic axon pathfinding defects in Tbr1, Gbx2, and Pax6 mutant mice: evidence that cortical and thalamic axons interact and guide each other. J Comp Neurol 447:8-17.

Hildebrand MS, Griffin NG, Damiano JA, Cops EJ, Burgess R, Ozturk E, Jones NC, Leventer RJ, Freeman JL, Harvey AS, Sadleir LG, Scheffer IE, Major H, Darbro BW, Allen AS, Goldstein DB, Kerrigan JF, Berkovic SF, Heinzen EL (2016) Mutations of the Sonic hedgehog pathway underlie hypothalamic hamartoma with gelastic epilepsy. Am J Hum Genet 99: 423-429.

Hill P, Wang B, Rüther U (2007) The molecular basis of palllister-hall associated polydactyly. Hum Mol Genet 16:2089-2096.

Ishibashi M, McMahon AP (2002) A Sonic hedgehog-dependent signaling rely regulates growth of diencephalic and mesencephalic primordia in the early mouse embryo. Development 129:4807-4819.

Izraeli S, Lowe LA, Bertness VL, Good DJ, Dorward DW, Kirsch IR, Kuehn MR (1999) The SIL gene is required for moue embryonic axial development and left-right specification. Nature 399:691-694.

Jeong Y, Dolson DK, Waclaw RR, Matise MP, Sussel L, Campbell K, Kaestner
KH, Epstein DJ (2011) Spatial and temporal requirements for Sonic hedgehog in the regulation of thalamic interneuron identity. Development 138:531-541.

Kakar N, Ahmad J, Morris-Rosendahl DJ, Altmüller J, Friedrich K, Barbi G, Nürnberg P, Kubisch C, Dobyns WB, Borck G (2015) STIL mutation causes autosomal recessive microcephalic lobar holoprosencephaly. Hum Genet 134:45-51.

Katarova Z, Sekerková G, Prodan S, Mugnaini E, Szabó G (2000) Domainrestricted expression of two glutamic acid decarboxylase genes in midgestation mouse embryos. J Comp Neurol 424:607-627.

Kiecker C, Lumsden A (2004) Hedgehog signaling from the ZLI regulates diecenphalic regional identity. Nat Neurosci 7:1242-1249.

Kim A, Savary C, Dubourg C, Carré W, Mouden C, Hamdi-Rozé H, Guyodo H, Douce JL; FREX Consortium; GoNL Consortium; Pasquier L, Flori E, Gonzales M, Bénéteau C, Boute O, Attié-Bitach T, Roume J, Goujon L, Akloul L, Odent S, et al. (2019) Integrated clinical and omics approach to rare diseases: novel genes and oligogenic inheritance in holoprosencephaly. Brain 142:35-49.

Kim EJ, Battiste J, Nakagawa Y, Johnson JE (2008) Ascl1 (Mash1) lineage cells contribute to discrete cell populations in CNS architecture. Mol Cell Neurosci 38:595-606.

Laclef C, Anselme I, Besse L, Catala M, Palmyre A, Baas D, Paschaki M, Pedraza M, Métin C, Durand B, Schneider-Maunoury S (2015) The role of primary cilia in corpus callosum formation is mediated by production of the GLI3 transcriptional repressor. Hum Mol Genet 24:4997-5014.

Lancaster MA, Schroth J, Gleeson JG (2011) Subcellular spatial regulation of canonical Wnt signalling at the primary cilium. Nat Cell Biol 13:700707.

Magnani D, Morlé L, Hasenpusch-Theil K, Paschaki M, Jacoby M, Schurmans S, Durand B, Theil T (2015) The ciliogenic transcription factor $\mathrm{Rfx} 3$ is required for the formation of the thalamocortical tract by regulating the patterning of prethalamus and ventral telencephalon. Hum Mol Genet 24:2578-2593.

Mahuzier A, Gaudé HM, Grampa V, Anselme I, Silbermann F, Leroux-Berger M, Delacour D, Ezan J, Montcouquiol M, Saunier S, SchneiderMaunoury S, Vesque C (2012) Dishevelled stabilization by the ciliopathy protein Rpgrip1l is essential for planar cell polarity. J Cell Biol 198: 927-940.

Martinez-Ferre A, Navarro-Garberi M, Bueno C, Martinez S (2013) Wnt signal specifies the intrathalamic limit and its organizer properties by regulating Shh induction in the alar plate. J Neurosci 33:3967-3980.

McNay DE, Pelling M, Claxton S, Guillemot F, Ang SL (2006) Mash1 is required for generic and subtype differentiation of hypothalamic neuroendocrine cells. Mol Endocrinol 20:1623-1632.

Miyashita-Lin EM, Hevner R, Wassarman KM, Martinez S, Rubenstein JL (1999) Early neocortical regionalization in the absence of thalamic innervation. Science 285:906-909.

Mouden C, de Tayrac M, Dubourg C, Rose S, Carré W, Hamdi-Rozé H, Babron MC, Akloul L, Héron-Longe B, Odent S, Dupé V, Giet R, David V (2015) Homozygous STIL mutation causes holoprosencephaly and microcephaly in two siblings. PLoS One 10:e0117418.

Mouden C, Dubourg C, Carré W, Rose S, Quelin C, Akloul L, Hamdi-Rozé H, Viot G, Salhi H, Darnault P, Odent S, Dupé V, David V (2016) Complex mode of inheritance in holoprosencephaly revealed by whole exome sequencing. Clin Genet 89:659-668.

Muenke M, Beachy PA (2001) Holoprosencephaly. In: Scriver CR et al., eds. The metabolic and molecular bases of inherited disease, Ed 8, pp 62036230. New York: McGraw-Hill.

Nakagawa Y, O'Leary DD (2001) Combinatorial expression patterns of LIM-homeodomain and other regulatory genes parcellate developing thalamus. J Neurosci 21:2711-2725.

Ocbina PJ, Tuson M, Anderson KV (2009) Primary cilia are not required for normal canonical Wnt signaling in the mouse embryo. PLoS One 4:e6839.

Paetau A, Salonen R, Haltia M (1985) Brain pathology in the meckel syndrome: a study of 59 cases. Clin Neuropathol 4:56-62.

Paetau A, Honkala H, Salonen R, Ignatius J, Kestilä M, Herva R (2008) Hydrolethalus syndrome: neuropathology of 21 cases confirmed by HYLS1 gene mutation analysis. J Neuropathol Exp Neurol 67:750-762.

Poretti A, Huisman TA, Scheer I, Boltshauser E (2011) Joubert syndrome and related disorders: spectrum of neuroimaging findings in 75 patients. AJNR Am J Neuroradiol 32:1459-1463.

Poretti A, Snow J, Summers AC, Tekes A, Huisman TAGM, Aygun N, Carson 
KA, Doherty D, Parisi MA, Toro C, Yildirimli D, Vemulapalli M, Mullikin JC; NISC Comparative Sequencing Program, Cullinane AR, Vilboux T, Gahl WA, Gunay-Aygun M (2017) Joubert syndrome: neuroimaging findings in 110 patients in correlation with cognitive function and genetic cause. J Med Genet 54:521-529.

Puelles E, Acampora D, Gogoi R, Tuorto F, Papalia A, Guillemot F, Ang SL, Simeone A (2006) Otx2 controls identity and fate of glutamatergic progenitors of the thalamus by repressing GABAergic differentiation. J Neurosci 26:5955-5964.

Puelles L, Martinez de la Torre M, Bardet S, Rubenstein JLR (2012) Hypothalamus. In: The mouse nervous system, Chap 8 (Watson C, Paxinos G, Puelles L, eds), pp 221-312. London, San Diego: Academic/Elsevier.

Reiter JF, Blacque OE, Leroux MR (2012) The base of the cilium: roles for transition fibres and the transition zone in ciliary formation, maintenance and compartmentalization. EMBO Rep 13:608-618.

Ribes V, Balaskas N, Sasai N, Cruz C, Dessaud E, Cayuso J, Tozer S, Yang LL, Novitch B, Marti E, Briscoe J (2010) Distinct sonic hedgehog signaling dynamics specify floor plate and ventral neuronal progenitors in the vertebrate neural tube. Genes Dev 24:1186-1200.

Roume J, Genin E, Cormier-Daire V, Ma HW, Mehaye B, Attie T, RazaviEncha F, Fallet-Bianco C, Buenerd A, Clerget-Darpoux F, Munnich A, Le Merrer M (1998) A gene for meckel syndrome maps to crhomosome 11q13. Am J Hum Genet 63:1095-1101.

Schwarz M, Cecconi F, Bernier G, Andrejewski N, Kammandel B, Wagner M, Gruss P (2000) Spatial specification of mammalian eye territories by reciprocal transcriptional repression of Pax2 and Pax6. Development 127:4325-4334.

Shi X, Garcia G 3rd, Van De Weghe JC, McGorty R, Pazour GJ, Doherty D, Huang B, Reiter JF (2017) Super-resolution microscopy reveals that disruption of ciliary transition-zone architecture causes Joubert syndrome. Nat Cell Biol 19:1178-1188.

Shimogori T, Lee DA, Miranda-Angulo A, Yang Y, Wang H, Jiang L, Yoshida AC, Kataoka A, Mashiko H, Avetisyan M, Qi L, Qian J, Blackshaw S (2010) A genomic atlas of mouse hypothalamic development. Nat Neurosci 13:767-775.

Shin SH, Kogerman P, Lindström E, Toftgárd R, Biesecker LG (1999) GLI3 mutations in human disorders mimic Drosophila cubitus interruptus protein functions and localization. Proc Natl Acad Sci U S A 96:2880-2884.

Simons M, Gloy J, Ganner A, Bullerkotte A, Bashkurov M, Krönig C, Schermer B, Benzing T, Cabello OA, Jenny A, Mlodzik M, Polok B, Driever W, Obara T, Walz G (2005) Inversin, the gene product mutated in nephronophthisis type II, functions as a molecular switch between Wnt signaling pathways. Nat Genet 37:537-543.

Stottmann RW, Tran PV, Turbe-Doan A, Beier DR (2009) Ttc21b is required to restrict sonic hedgehog activity in the developing mouse forebrain. Dev Biol 335:166-178.
Stoykova A, Gruss P (1994) Roles of pax-genes in developing and adult brain as suggested by expression patterns. J Neurosci 14:1395-1412.

Stoykova A, Fritsch R, Walther C, Gruss P (1996) Forebrain patterning defects in small eye mutant mice. Development 122:3453-3465.

Struchtrup A, Wiegering A, Stork B, Rüther U, Gerhardt C (2018) The ciliary protein RPGRIP1L governs autophagy independently of its proteasome-regulating function at the ciliary base in mouse embryonic fibroblasts. Autophagy 14:567-583.

Szabó NE, Zhao T, Zhou X, Alvarez-Bolado G (2009a) The role of sonic hedgehog of neural origin in thalamic differentiation in the mouse. J Neurosci 29:2453-2466.

Szabó NE, Zhao T, Cankaya M, Theil T, Zhou X, Alvarez-Bolado G (2009b) Role of neuroepithelial sonic hedgehog in hypothalamic patterning. J Neurosci 29:6989-7002.

Vierkotten J, Dildrop R, Peters T, Wang B, Rüther U (2007) Ftm is a novel basal body protein of cilia involved in Shh signaling. Development 134:2569-2577.

Vue TY, Aaker J, Taniguchi A, Kazemzadeh C, Skidmore JM, Martin DM, Martin JF, Treier M, Nakagawa Y (2007) Characterization of progenitor domains in the developing mouse thalamus. J Comp Neurol 505:73-91.

Vue TY, Bluske K, Alishahi A, Yang LL, Koyano-Nakagawa N, Novitch B, Nakagawa Y (2009) Sonic hedgehog signaling controls thalamic progenitor identity and nuclei specification in mice. J Neurosci 29:44844497.

Wiegering A, Dildrop R, Kalfhues L, Spychala A, Kuschel S, Lier JM, Zobel T, Dahmen S, Leu T, Struchtrup A, Legendre F, Vesque C, SchneiderMaunoury S, Saunier S, Rüther U, Gerhardt C (2018) Cell type-specific regulation of ciliary transition zone assembly in vertebrates. EMBO J 37:e97791.

Willaredt MA, Hasenpusch-Theil K, Gardner HA, Kitanovic I, HirschfeldWarneken VC, Gojak CP, Gorgas K, Bradford CL, Spatz J, Wölfl S, Theil T, Tucker KL (2008) A crucial role for primary cilia in cortical morphogenesis. J Neurosci 28:12887-12900.

Willaredt MA, Tasouri E, Tucker KL (2013) Primary cilia and forebrain development. Mech Dev 130:373-380.

Zeltser LM (2005) Shh-dependent formation of the ZLI is opposed by signals from the dorsal diencephalon. Development 132:2023-2033.

Zhang Y, Alvarez-Bolado G (2016) Differential developmental strategies by sonic hedgehog in thalamus and hypothalamus. J Chem Neuroanat 75:20-27.

Zhao L, Zevallos SE, Rizzoti K, Jeong Y, Lovell-Badge R, Epstein DJ (2012) Disruption of SoxB1 dependent sonic hedgehog expression in the hypothalamus causes septo-optic dysplasia. Dev Cell 22:585-596.

Zhou CJ, Pinson KI, Pleasure SJ (2004) Severe defects in dorsal thalamic development in low-density lipoprotein receptor-related protein- $6 \mathrm{mu}-$ tants. J Neurosci 24:7632-7639. 